\title{
THE STRUCTURE, ORIGIN, AND EVOLUTION OF INTERSTELLAR HYDROCARBON GRAINS
}

\author{
J. E. Chiar ${ }^{1}$, A. G. G. M. Tielens ${ }^{2}$, A. J. Adamson ${ }^{3}$, And A. Ricca ${ }^{1}$ \\ ${ }^{1}$ SETI Institute, Carl Sagan Center, 189 Bernardo Avenue, Mountain View, CA 94043, USA; jchiar@ seti.org, Alessandra.Ricca-1.nasa.gov \\ ${ }^{2}$ Leiden Observatory, P.O. Box 9513, NL-2300 RA Leiden, The Netherlands; tielens@ strw.leidenuniv.nl \\ ${ }^{3}$ Gemini Observatory, Northern Operations Center, 670 North A'ohoku Place, Hilo, HI 96729, USA; aadamson@ gemini.edu \\ Received 2012 July 12; accepted 2013 April 23; published 2013 May 24
}

\begin{abstract}
Many materials have been considered for the carrier of the hydrocarbon absorption bands observed in the diffuse interstellar medium (ISM). In order to refine the model for ISM hydrocarbon grains, we analyze the observed aromatic $(3.28,6.2 \mu \mathrm{m})$ and aliphatic $(3.4 \mu \mathrm{m})$ hydrocarbon absorption features in the diffuse ISM along the line of sight toward the Galactic center Quintuplet Cluster. Observationally, $s p^{2}$ bonds can be measured in astronomical spectra using the $6.2 \mu \mathrm{m}$ CC aromatic stretch feature, whereas the $3.4 \mu \mathrm{m}$ aliphatic feature can be used to quantify the fraction of $s p^{3}$ bonds. The fractional abundance of these components allows us to place the Galactic diffuse ISM hydrocarbons on a ternary phase diagram. We conclude that the Galactic hydrocarbon dust has, on average, a low $\mathrm{H} / \mathrm{C}$ ratio and $s p^{3}$ content and is highly aromatic. We have placed the results of our analysis within the context of the evolution of carbon dust in the ISM. We argue that interstellar carbon dust consists of a large core of aromatic carbon surrounded by a thin mantle of hydrogenated amorphous carbon (a-C:H), a structure that is a natural consequence of the processing of stardust grains in the ISM.
\end{abstract}

Key words: astrochemistry - dust, extinction - ISM: lines and bands - ISM: molecules - techniques: spectroscopic

Online-only material: color figures

\section{INTRODUCTION}

Carbonaceous dust is the carrier of a number of infrared absorption features: specifically at $\sim 3.4,6.8$, and $7.2 \mu \mathrm{m}$, as well as the strong UV resonance at $2175 \AA$. In the Milky Way, absorption bands due to the $\mathrm{CH}$ stretching $(3.4 \mu \mathrm{m})$ and deformation $(6.85,7.25 \mu \mathrm{m})$ modes of methyl $\left(\mathrm{CH}_{3}\right)$ and methylene $\left(\mathrm{CH}_{2}\right)$ groups in aliphatic hydrocarbon materials are detected along lines of sight that probe the local diffuse interstellar medium (ISM) within $3 \mathrm{kpc}$ of the Sun (Adamson et al. 1990; Sandford et al. 1991; Pendleton et al. 1994; Whittet et al. 1997) and along the line of sight toward the Galactic center (Butchart et al. 1986; McFadzean et al. 1989; Sandford et al. 1991; Pendleton et al. 1994; Tielens et al. 1996; Chiar et al. 2000, 2002). The $3.4 \mu \mathrm{m}$ absorption band has also been detected in one young planetary nebula, AFGL 618 (Lequeux \& Jourdain de Muizon 1990; Chiar et al. 1998). In recent years, groundbased, Spitzer Space Telescope, and AKARI observations have revealed that absorption bands of aliphatic hydrocarbons are also common in dense and dusty galactic nuclei of Seyfert and UltraLuminous InfraRed Galaxies (ULIRGs; Imanishi 2000; Spoon et al. 2001, 2004; Mason et al. 2004; Imanishi et al. 2006; Armus et al. 2007; Dartois \& Muñoz-Caro 2007; Kondo et al. 2012). In the Galactic diffuse ISM, absorption at $\sim 3.3 \mu \mathrm{m}$ due to the $\mathrm{C}-\mathrm{H}$ stretch in aromatics has been discussed by Chiar et al. (2000, 2002; cf. Section 3.1). An absorption band due to the $\mathrm{CC}$ stretch in aromatic hydrocarbons has been detected in the diffuse ISM in one extragalactic source; however, the weaker corresponding $\mathrm{CH}$ stretch eluded detection (Dartois et al. 2007).

A wide variety of materials have been considered as the carrier of these interstellar hydrocarbon bands mainly differing in their production methods (cf. Pendleton \& Allamandola 2002). What these materials generally have in common is that they contain aromatic and aliphatic functional groups. The beststudied material which also provides the best fit to the IR observations is hydrogenated amorphous hydrocarbon $(\mathrm{a}-\mathrm{C}: \mathrm{H}$;
Duley 1994; Duley et al. 1998; Mennella et al. 2002). Laboratory studies have shown that hydrogenated amorphous carbon materials may be responsible for the $2175 \AA$ feature (Mennella et al. 1996; Schnaiter et al. 1999; Gadallah et al. 2011).

In this paper, we combine previously published spectra of the Galactic center Quintuplet sources from the Infrared Space Observatory (ISO; Chiar et al. 2000) and previously unpublished 3.02-3.65 $\mu \mathrm{m}$ spectra from UKIRT-CGS4 of six individual Quintuplet Cluster sources in order to investigate the nature and structure of the carbonaceous dust in the diffuse ISM. In Section 2, we describe the previously published ISO spectra and new UKIRT spectra. In Section 3, we describe the characteristics and assignments of the absorption features as well as the resulting column densities of the $\mathrm{CC}$ and $\mathrm{CH}$ vibrational modes. Based on the measurements and calculations presented in Section 3, we deduce the structure of the hydrocarbon grains in Section 4. Finally, we conclude with a discussion of the evolution of carbonaceous grains in Section 5 and summarize our results in Section 6.

\section{OBSERVATIONS AND DATA REDUCTION}

Using the United Kingdom Infrared Telescope (UKIRT) and the cooled grating spectrometer, CGS4, we obtained spectra in the Quintuplet region using the 1 pixel 0'.61 slit, effectively sampling the dust with higher spatial resolution than possible with ISO-SWS. With UKIRT-CGS4, we obtained spectra of GCS3-I, II, III, and IV; GCS4; and MGM5 from 2.9 to $3.64 \mu \mathrm{m}$ on UT 2000-06-21. The 40 lines $\mathrm{mm}^{-1}$ grating and the 1 pixel (0'61) slit resulted in a resolving power of $\lambda / \Delta \lambda \sim 1400$. Wavelength calibration was achieved using an argon lamp. Telluric cancellation was carried out by observing early-type standard stars within 0.01-0.02 airmass of each of the targets. The resulting ratioed spectra are shown in Figure 1 (left panels) in units of ratioed flux. Shown in the same figure are the thirdorder polynomials that were used to fit the continuum for each spectrum. The resulting optical depth spectra are shown in the 

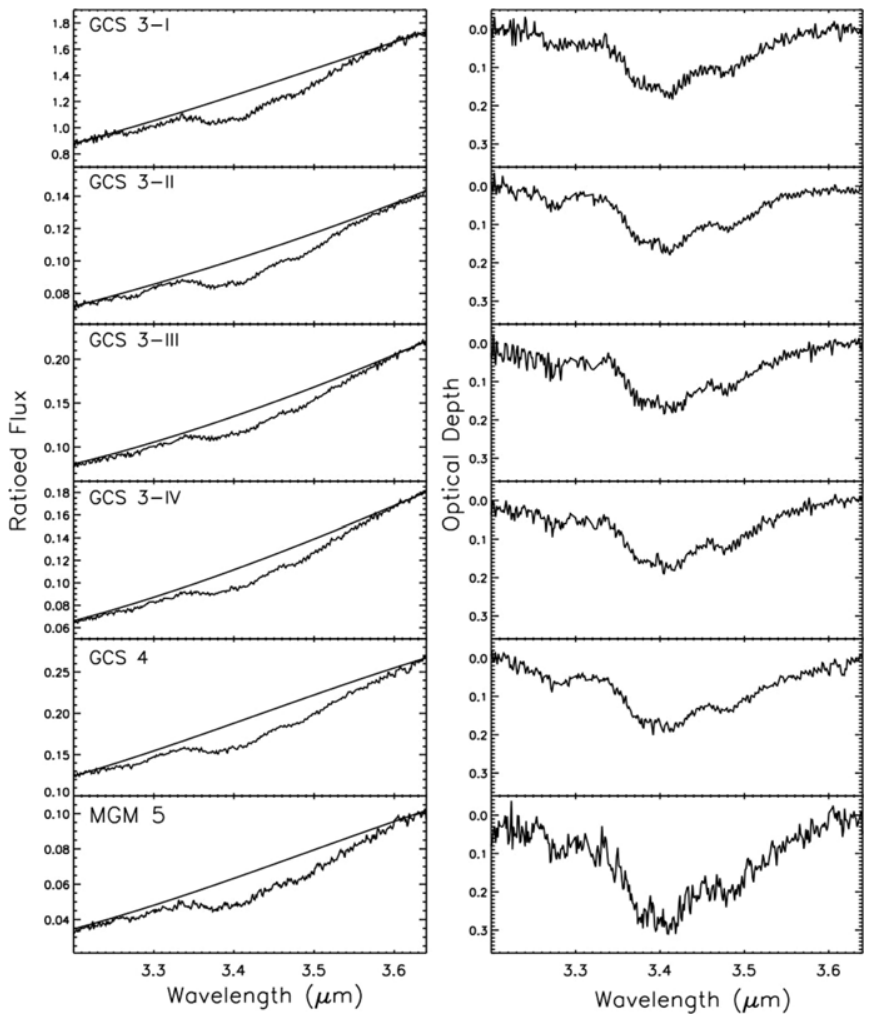

Figure 1. Ratioed flux spectra with fitted third-order polynomial (left panels) and corresponding resulting optical depth spectra (right panels).

right-hand panel of Figure 1. The $I S O$-SWS 2 to $13 \mu \mathrm{m}$ spectrum of the Quintuplet region was previously analyzed by Chiar et al. (2000). The ISO-SWS beam was large at $14^{\prime \prime} \times 20^{\prime \prime}$, so the observations that were centered on GCS 3 I included GCS 3-I, II, III, and IV. The $6 \mu \mathrm{m}$ region of the previously published $I S O$-SWS spectrum is shown in Figure 2.

The Quintuplet region likely suffers from differential extinction (e.g., Figer et al. 1999), however, given the uniformity of the profile shape and depth of the hydrocarbon absorption features presented here, we are confident that the dust properties and extinction have negligible variation for these particular lines of sight. With this in mind, we averaged the spectra from the individual lines of sight to create a high signal-to-noise mean spectrum. The resulting high $\mathrm{S} / \mathrm{N}$ spectrum allows us to more accurately measure the substructure of the hydrocarbon components. To compute the weighted average, we first calculated the standard deviation at each wavelength across the spectrum with respect to a smooth function that follows the feature shape. The average standard deviation was then used to weight each spectrum in computing the mean profile that is shown in Figure 2.

\section{THE ASTRONOMICAL HYDROCARBON SPECTRUM}

\subsection{The Carrier(s) of the Absorption Features}

The 3.2 to $3.65 \mu \mathrm{m}$ astronomical spectrum contains both aromatic and aliphatic (and, likely, olefinic) absorption bands. The 5.6 to $6.5 \mu \mathrm{m}$ region contains olefinic and aromatic components. To be consistent with the analysis in the literature of the hydrocarbon bands, we uses Gaussian profiles to decompose the bands (e.g., Gielen et al. 1996; Ristein et al. 1998). In this way, we avoid assuming that the bands have strong wings such as Lorentzian and Drude shapes (see discussion in Jones 2012c). In order to disentangle these components, we carried out

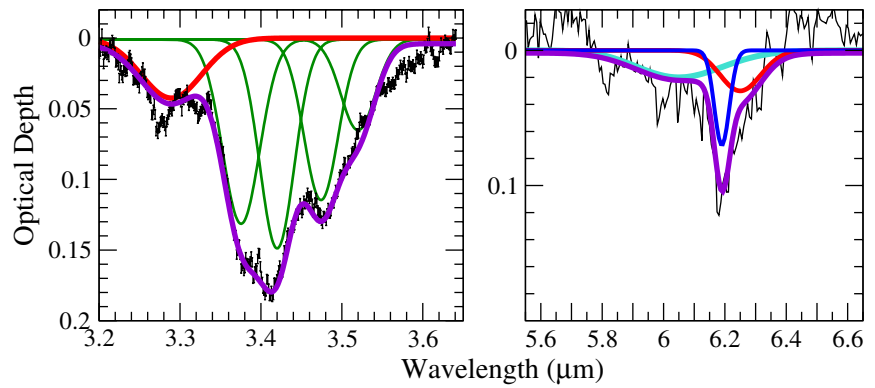

Figure 2. Left panel: optical depth spectrum computed from the weighted average of the observed spectra for the six quintuplet lines of sight. Of the five fitted Gaussians shown, four (green lines) are representative of the aliphatic hydrocarbon component, and one (red line) is representative of the aromatic component. The narrow feature that dips below the (red) aromatic band may be an olefinic component; this component has been omitted from the Gaussian fit. The co-added result of the fitted Gaussians is represented by the purple line. Right panel: optical depth spectrum of the $6 \mu \mathrm{m}$ region. Aromatic (red) and olefinic (dark blue) components are shown. The absorption feature represented by the light blue Gaussian is unidentified, but could be due to carbonyl groups. The co-added result of the fitted Gaussians is represented by the purple line. See text for description.

(A color version of this figure is available in the online journal.)

independent Gaussian fits to the 3.4 and $6.2 \mu \mathrm{m}$ observed absorption features ${ }^{4}$ (Figure 2). The (mean) diffuse ISM $3.4 \mu \mathrm{m}$ spectrum is decomposed into five Gaussians representing the $s p^{2}$ $\mathrm{CH}, s p^{3} \mathrm{CH}_{3}$ and $\mathrm{CH}_{2}$ asymmetric, and $s p^{3} \mathrm{CH}_{3}$ and $\mathrm{CH}_{2}$ symmetric modes (Figure 2, left panel). The central wavelengths and widths of these components were allowed to vary within limits determined by various laboratory experiments that produced hydrocarbon films (Heitz et al. 1998; Gielen et al. 1996; Dischler 1983; Ristein et al. 1998). The Gaussians that peak longward of $3.3 \mu \mathrm{m}$ represent the aliphatic hydrocarbon bands. Shortward of the aliphatic bands is a broad band centered at $3.3 \mu \mathrm{m}$ (red curve in Figure 2 discussed below), which we attribute to aromatics. Superposed on this band is a relatively narrow $\left(\sim 25 \mathrm{~cm}^{-1}\right)$ band centered at $3.28 \mu \mathrm{m}$ which we attribute to the olefinic $\mathrm{CH}$ mode. This narrow band (which dips below the red smooth Gaussian curve that represents the broader aromatic band) is omitted from the fitting procedure.

In the Quintuplet line of sight, there is also dense cloud material as evidenced by the observed $\mathrm{H}_{2} \mathrm{O}$-ice absorption feature at $3 \mu \mathrm{m}$ (Chiar et al. 2000). Chiar et al. (2000) used the observed $3 \mu \mathrm{m} \mathrm{H} \mathrm{H}_{2} \mathrm{O}$-ice feature to constrain and subtract the contribution of ice absorption in the $6 \mu \mathrm{m}$ region in the Quintuplet spectrum. We use that ice-subtracted $6 \mu \mathrm{m}$ spectrum here. The resulting ice-subtracted $6 \mu \mathrm{m}$ spectrum closely resembles previously published ice-free $6 \mu \mathrm{m}$ spectra (cf. Chiar et al. 2000), lending confidence that there is no residual ice absorption in the Quintuplet spectrum (Chiar et al. 2000, their Figure 8). Experimental studies of hydrocarbon films show that the $6 \mu \mathrm{m}$ hydrocarbon absorption feature is likely due to both aromatic and olefinic modes (Wexler 1967; Bourée et al. 1996). These studies show that the olefinic band is narrower and peaks at shorter wavelengths $\left(\lambda_{0} \sim 6.1 \mu \mathrm{m}\right)$ than the aromatic CC mode which peaks at $\sim 6.3 \mu \mathrm{m}$ (Bourée et al. 1996; Choi et al. 2001). While we use these central wavelengths to guide the decomposition of the astronomical $6 \mu \mathrm{m}$ spectrum, it is apparent that a third component at $\sim 6.0 \mu \mathrm{m}$ is present (light blue curve). This absorption may be due to carbonyl groups which absorb in this region (Wexler 1967). The $6 \mu \mathrm{m}$ spectrum with the best fitted

\footnotetext{
4 The Gaussian fits were not carried out simultaneously over the 3 and $6 \mu \mathrm{m}$ regions.
} 
Table 1

Fit Parameters for Diffuse ISM Hydrocarbon Feature

\begin{tabular}{lccccc}
\hline \hline Mode & $\begin{array}{c}\text { Central Wavelength } \\
(\mu \mathrm{m})\end{array}$ & $\begin{array}{c}\Delta \lambda \\
(\mu \mathrm{m})\end{array}$ & $\begin{array}{c}\Delta v \\
\left(\mathrm{~cm}^{-1}\right)\end{array}$ & $\begin{array}{c}\text { Integrated Area } \\
\left(\mathrm{cm}^{-1}\right)\end{array}$ & $\begin{array}{c}\text { Absorption Strength } \\
\left(\times 10^{-18} \mathrm{~cm} \mathrm{group}\right.\end{array}$ \\
\hline $\mathrm{CH}_{3}\left(s p^{3}\right)$ asym. & 3.376 & 0.05 & 47.2 & 6.55 & $\begin{array}{c}\text { Column Density } \\
\left(\times 10^{18} \mathrm{group} \mathrm{cm}^{-2}\right)\end{array}$ \\
$\mathrm{CH}_{2}\left(s p^{3}\right)$ asym. & 3.420 & 0.05 & 42.8 & 6.74 & 0.27 \\
$\mathrm{CH}_{3}\left(s p^{3}\right)$ sym. & 3.474 & 0.05 & 41.0 & 4.96 & 15.2 \\
$\mathrm{CH}_{2}\left(s p^{3}\right)$ sym. & 3.520 & 0.05 & 40.4 & 2.76 & 23.7 \\
$\mathrm{CH}\left(s p^{2}\right)^{\mathrm{a}}$ & 3.289 & 0.09 & 81.8 & 0.37 & 0.21 \\
$\mathrm{CC}\left(s p^{2}\right)^{\mathrm{b}}$ & $6.19,6.25$ & $0.06,0.16$ & 15,40 & $1.05,1.20$ & 0.19 \\
\hline
\end{tabular}

Notes.

a Values are for the aromatic mode only.

b Values are for olefinic and aromatic modes, respectively. Absorption strength is for the aromatic band; column density is the sum of both bands using the aromatic strength. See text for details.

c Absorption strength for $\mathrm{CC}$ mode is in units of $\mathrm{cm}$ atom ${ }^{-1}$.

three Gaussian curves is shown in Figure 2 (right panel). The parameters (central wavelength, full width at half-maximum, and integrated area) for the aromatic and aliphatic modes are listed in Table 1.

In the 3.2 to $3.65 \mu \mathrm{m}$ region, the fitting procedure described above results in a broad, $\Delta \nu \sim 80 \mathrm{~cm}^{-1}$, aromatic band centered at $3.289 \mu \mathrm{m}$. A much narrower absorption feature centered at $3.28 \mu \mathrm{m}$ toward the Galactic center was discussed by Pendleton et al. (1994), but these authors state that the analysis of the feature was made difficult by incomplete cancellation of telluric and standard star features, so identification with an interstellar feature was left as an open question. A similarly narrow feature centered at $3.28 \mu \mathrm{m}\left(\Delta v \sim 25 \mathrm{~cm}^{-1}\right)$ was detected by Chiar et al. (2000) toward the Quintuplet Cluster line of sight; this feature, which we also detect in the spectra presented here (most apparent in the median spectrum in Figure 2, left panel), is likely the olefinic $\mathrm{C}-\mathrm{H}$ mode of hydrogenated amorphous carbon grains. Chiar et al. (2002) find a broad feature $\left(\sim 100 \mathrm{~cm}^{-1}\right)$ centered around $3.3 \mu \mathrm{m}$ in their analysis of six spectra along the line of sight toward the Galactic center central cluster. They found a strong correlation between the $3.4 \mu \mathrm{m}$ aliphatic feature and the $3.3 \mu \mathrm{m}$ broad aromatic feature, supporting our assignment of the $3.3 \mu \mathrm{m}$ band to a component of the dust. While the $\mathrm{CH}$ stretch in polycyclic aromatic hydrocarbons (PAHs) is a logical candidate for this absorption, typical astronomical $3.3 \mu \mathrm{m}$ PAH emission features have much narrower widths ( $\sim 30 \mathrm{~cm}^{-1}$; Sellgren 2001 and references therein). Although temperature effects can lead to broadening of the profile because of higher $J$ levels being populated at higher temperature (Joblin et al. 1995), a width near $100 \mathrm{~cm}^{-1}$ would imply temperatures greater than $\sim 2000 \mathrm{~K}$, much too high for the diffuse ISM, where the temperature of dust grains is constrained to 10-25 K (Mathis et al. 1983). Brooke et al. (1999) observe a similarly broad $\left(74 \mathrm{~cm}^{-1}\right)$ absorption feature that is blue-shifted $\left(\lambda_{0}=3.25 \mu \mathrm{m}\right)$ compared to the diffuse ISM feature in several lines of sight toward dense clouds, where the feature is attributed to cold aromatic hydrocarbons.

As the sight lines toward the Quintuplet sources show ice absorption (Chiar et al. 2000; Moneti et al. 2001), we also consider PAHs embedded in an $\mathrm{H}_{2} \mathrm{O}$-ice matrix. PAHs embedded in $\mathrm{H}_{2} \mathrm{O}$-ice have broader bands compared to PAHs in inert matrices, but the bands are still narrow $\left(\Delta v \sim 10-20 \mathrm{~cm}^{-1}\right.$ for the $\mathrm{CH}$ stretch band and $\Delta v \sim 4-10 \mathrm{~cm}^{-1}$ for the $\mathrm{CC}$ stretch band; Bernstein et al. 2005) compared with the astronomical absorption features discussed here. Thus, PAHs (icy or otherwise) are not strong candidates for the diffuse ISM absorption features. It is more likely that the aromatic absorption features are caused by a component of the hydrocarbon dust, linked to the aliphatics.

\subsection{Column Densities}

To better understand the nature of the diffuse ISM hydrocarbons, we calculate the column densities of the aromatic and aliphatic components. We then use these column densities to calculate the relative abundances of the components which, in turn, define the hydrocarbon structure (i.e., the placement of the diffuse ISM data point on the ternary phase diagram). The intrinsic strength of the absorption components is needed in order to calculate the column density of the absorber. There has been extensive discussion in the literature on the intrinsic strength of solid hydrocarbon absorption features (Tanaka et al. 1990; Gielen et al. 1996; Furton et al. 1999; Choi et al. 2001; Robertson 2002). The independent measurements of IR absorption strength, $\sigma_{\text {group }}$, and H-content can be calculated for each bond group by

$$
\sigma_{\text {group }}=\omega p / A_{\text {atom }},
$$

where $\omega$ is the band frequency $\left(\mathrm{cm}^{-1}\right)$ and $p$ is equal to 1,2 , 3 in $\mathrm{CH}_{p}$. The proportionality constant, $A_{\text {atom }}$ (per $\mathrm{H}$ atom for the $\mathrm{CH}$ modes), takes into account the effects of the dielectric medium (the carbonaceous matrix) on the intrinsic strength of the bands and has been determined from laboratory studies of hydrocarbon films. We assume that each bonding type (i.e., $s p^{y} \mathrm{CH}_{x}$ ) has a different proportionality constant that is the same for all hydrocarbon films. For $s p^{3}$ modes, these constants are $A_{\text {atom }}=3.85 \times 10^{21}$ atoms cm$~^{-2}$ for $\mathrm{CH}_{2}$ modes and $3.65 \times 10^{21}$ atoms cm${ }^{-2}$ for $\mathrm{CH}_{3}$ modes (Tanaka et al. 1990). The intrinsic strength for the $s p^{2} \mathrm{CH}$ mode is determined from the intensity ratios per $\mathrm{CH}$ bond: $s p^{3} \mathrm{CH}: s p^{3} \mathrm{CH}_{2}: s p^{2} \mathrm{CH}: s p^{1} \mathrm{CH}=$ 1:10:3.4:3.4 (Gielen et al. 1996), which translates to $s p^{2} \mathrm{CH}: s p^{3} \mathrm{CH}_{2}=3.4: 20$ per group. For the $\mathrm{CC}$ mode, we use the absorption spectrum measured by Choi et al. (2001) for a$\mathrm{C}: \mathrm{H}$ films to compute an absorption strength, assuming a density of $2 \mathrm{~g} \mathrm{~cm}^{-3}$, typical for amorphous carbon (Robertson 2002). The absorption strengths (Table 1) are in units of $\mathrm{cm} \mathrm{atom}^{-1}$ for the $\mathrm{CC}$ mode and $\mathrm{cm}_{\text {group }}{ }^{-1}$ for the $\mathrm{CH}$ modes. The strengths in Table 1 are approximately a factor of two larger than those used in previous work (e.g., Sandford et al. 1991; Pendleton et al. 1994; Dartois et al. 2007). The previously adopted values assumed PAHs (benzene derivatives) for the aromatic bands, saturated aliphatic hydrocarbons (e.g., hexane) for the aliphatic 
material, and did not take into account the effects of the carbon matrix on the band strength. The latter correction factor is, of course, imperative for a proper comparison to laboratory studies on the structure of hydrocarbon films and for a correct placement on the ternary diagram relative to laboratory studies of well-characterized materials.

Computed column densities for $\mathrm{CC}$ and $\mathrm{CH}$ modes in the diffuse ISM are based on the integrated areas determined by the Gaussian fit shown in Figure 2 (Table 1). Column densities are calculated in the usual way:

$$
N=\tau \Delta \nu / \sigma_{\text {group }}\left(\mathrm{cm}^{-2}\right)
$$

where $\tau \Delta v$ is the integrated area of the individual hydrocarbon component as measured by the fitted Gaussian and $\sigma_{\text {group }}$ is the intrinsic strength per group.

\section{CARBON STRUCTURE}

Carbonaceous materials show a great diversity in properties because the four valence electrons of carbon can exist in three hybridization states- $s p^{3}, s p^{2}$, and $s p^{1}$ —and four structural states, aliphatic (alkane), olefinic (alkene), aromatic, and alkyne (Robertson 2002). In $s p^{3}$ hybridization, all four electrons are bonded through covalent $\sigma$ orbitals to neighboring atoms which is the basis of a diamond-like network. In $s p^{2}$ hybridization, three of the electrons make such directed $\sigma$ bonds forming a plane, while the fourth electron is in a $\pi$-orbital. The $\pi$ electrons of adjacent carbon atoms can conjugate, forming weaker $\pi$ bonds perpendicular to this plane, resulting in a graphitic structure or quasi-linear olefinic chains. These olefinic components can be important structural bonding units, in addition to the aliphatics, especially at low hydrogen content (Jones 2012a). In the $s p^{1}$ hybridization, only two electrons are in $\sigma$ bonds leading to linear chains, and the other two electrons are in $\pi$ bonds.

The physical properties of a-C:H materials are intimately linked to their microscopic structure (Robertson 2002). In an astrophysical context, these materials have been studied from a theoretical point of view (Jones 1990, 2012a, 2012b, 2012c) and relevant laboratory studies have been performed (Duley \& Williams 1983; Duley 1984, 1994). Hydrogenated amorphous carbon films consist of a strongly cross-linked threedimensional network where aromatic clusters and/or aliphatic chains are bonded through aliphatic structures. The tetrahedrally bonded carbon ( $s p^{3}$ hybrids) leads to a three-dimensional network that provides the material hardness, while the aromatic clusters ( $s p^{2}$ hybrids) in the network set the optical properties because they control the optical gap of the material. The various forms of carbonaceous materials are then distinguished by their $\mathrm{H}$ content that is directly linked to $s p^{3} / s p^{2}$ ratio. Conversely, by measuring the fraction of $s p^{2}, s p^{3}$, and $\mathrm{H}$ in the material, the physical properties can be determined.

Following, e.g., Robertson (2002) and Jacob \& Möller (1993), the structural characteristics of a-C:H films can be visualized through a ternary diagram that plots the $s p^{2}, s p^{3}$, and $\mathrm{H}$ fraction, which are normalized so that their sum is unity. This ternary diagram (Figure 3) is a triangle where the apexes represent (counterclockwise from the top) $s p^{3}, s p^{2}$, and $\mathrm{H}$ content. Consider first the $s p^{3}$ content (top apex): horizontal lines in the diagram represent constant $s p^{3}$ fractions with unity at the top and zero at the bottom. The arrow on the right-hand side of the triangle indicates the direction of increasing $s p^{3}$ fraction. Similarly, the $s p^{2}$ fraction is given by considering the left-hand

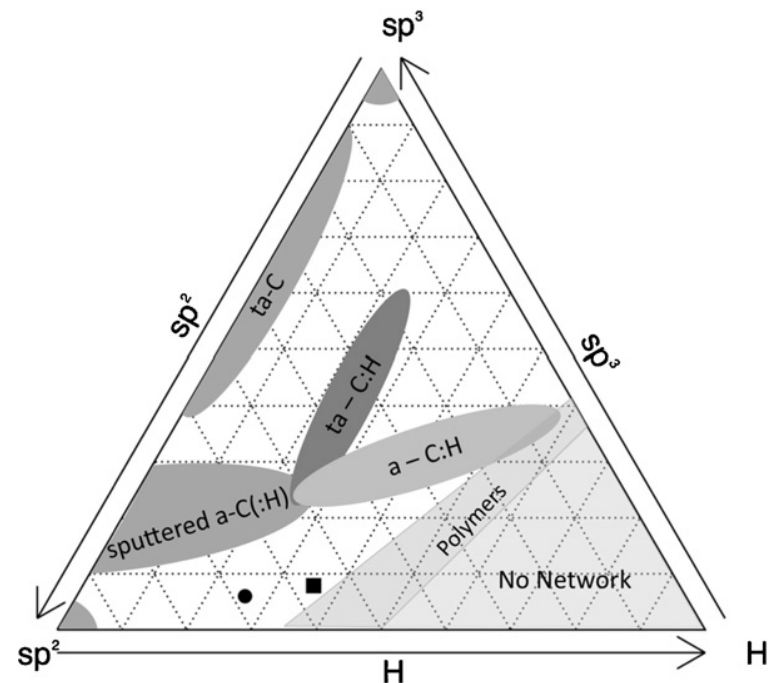

Figure 3. Ternary diagram for hydrocarbon materials. Placement of the various forms of hydrocarbons on this diagram is discussed in detail by Robertson (2002) and Jacob \& Möller (1993). Glassy carbon materials such as soot, chars, and graphite have pure $s p^{2}$ bonds and are located in the lower left of the diagram. Diamond-like carbon lies at the apex of the diagram because of its pure $s p^{3}$ bonds. The locations of sputtered amorphous carbon (a-C), tetrahedral amorphous carbon (ta-C), tetrahedral hydrocarbon (ta-C:H), and amorphous hydrocarbon (a-C:H) are also shown. The lower right-hand corner of the diagram represents the region where no stable hydrocarbon films can be formed. The loci of the GC diffuse ISM hydrocarbons as determined by the absorption strengths determined in this work and by Ristein et al. (1998) are represented by the circle and square, respectively.

corner as the apex and the right-hand side as the base. Constant $s p^{2}$ fractions are again parallel to the base with unity in the apex (left-hand corner) and zero on the base. The H-content is given by the right-hand corner as the apex and the left-hand side as the base. In a similar way, constant $\mathrm{H}$ fraction is given by lines parallel to this base with unity in the right-hand corner and zero at the base. Pure graphite and pure diamond are located in the $s p^{2}$ and $s p^{3}$ apexes, respectively. The shaded right corner of the diagram represents the region where no stable hydrocarbon films can form. At the edge of this "no network" region, the hydrogen content is low enough for polymers to form.

The characterization of different carbonaceous material and their location in the ternary diagram has been described by Ferrari \& Robertson (2000) and Robertson (2002). In an astrophysical context, the ternary diagram has been introduced by Dartois et al. (2007). Along the $s p^{2}-s p^{3}$ side of the triangle, the fraction of $s p^{3}$-bonded $\mathrm{C}$ increases and disorder increases because the hexagonal graphitic structure is broken up by the introduction of defects. In a-C material, there are some $s p^{3}$ sites and the amorphous carbon is more disordered because of the incorporation of odd-membered and distorted rings (Figure 3). For ta-C material, there is more $s p^{3}$ content and the $s p^{2}$ configuration changes from rings to short (olefinic) chains (Figure 3). In a-C:H, the $s p^{3}$ sites form a continuous network of $\mathrm{CC}$ bonds and the $s p^{2} \mathrm{C}$ are arranged as small clusters. For low $\mathrm{H}$ content, the $s p^{2} \mathrm{C}$ is aromatic in nature while for high $\mathrm{H}$ content, olefinic chains dominate. A large part of the $s p^{3}$ bonding in a-C:H is due to saturation of bonding with $\mathrm{H}$ and, indeed, the $s p^{3}$ fraction is directly linked to the hydrogen content. The $\mathrm{H}$ content in ta-C:H is low and hence, unlike for a-C:H there is no polymeric regime. The ta-C:H films with high $s p^{3}$ fraction $(\sim 0.60)$ and low $\mathrm{H}$ content $(\sim 0.25)$ have a high propensity of $\mathrm{CC}$ bonds and are characterized by a tetrahedral $\mathrm{C}$ network. 
Table 2

Hydrogen and Carbon Content in ISM Grains

\begin{tabular}{lcc}
\hline \hline Atom & Mode & $\begin{array}{c}\text { Abundance } \\
\left(\times 10^{-4}\right)\end{array}$ \\
\hline Carbon & $s p^{2}$ & 1.49 \\
& $s p^{3}$ & 0.13 \\
Hydrogen & $s p^{2}$ & 0.26 \\
& $s p^{3}$ & 0.31 \\
\hline
\end{tabular}

Typical carbon polymers such as polystyrene and polyvinyl have a very high $\mathrm{H}$-content and little to no cross linking of the carbonchains. Those are located along the $80 \% \mathrm{H}$ content line. Simple molecular solids are located beyond this line in this corner of carbon films. Solids consisting of PAH molecules are located along the base line connecting the $s p^{2}$ and $\mathrm{H}$ apexes while pure alkane solids are along the line connecting $s p^{3}$ and $\mathrm{H}$ apexes.

Given this propensity of bonding and compositional variations, many different carbonaceous materials have been produced using a variety of techniques and characterized by different analytical methods. These materials do not uniformly cover the ternary diagram but, rather, are concentrated in specific regions, basically because these production methods result in materials with systematic variations in their structure. These regions are indicated in Figure 3. Conversely, we can determine the properties of a material by locating it on this ternary diagram. Table 1 summarizes the observed characteristics of the different IR bands that can be used to determine $s p^{2}, s p^{3}$, and $\mathrm{H}$ content. While some of these have been reported before in other studies, some-notably the aromatic $\mathrm{H}$ and aromatic $\mathrm{C}$ - have only been measured in a few and very special environments. Here, we discuss all available constraints on the three material characteristics that span up the ternary diagram for carbon dust in the diffuse ISM.

The abundance of elemental carbon (and hydrogen) involved in the aliphatic and aromatic absorption features cannot be accurately calculated because, for the Galactic center, the total hydrogen column in not known. Assuming $N(\mathrm{H}) / A_{V}$ appropriate for the solar neighborhood, and $A_{V} \sim 29 \mathrm{mag}$ (estimated from near-IR color excess by Figer et al. 1999), we deduce the $\mathrm{C}$ and $\mathrm{H}$ abundances listed in Table 2. We emphasize that the abundances listed in Table 2 should only be considered indicative rather than firm measurements. Strictly speaking, we cannot assume the $N(\mathrm{H}) / A_{V}$ ratio which is derived from local UV studies, since the dust-to-gas fraction in the Galactic center region is likely to be much higher than the local value in view of the Galactic elemental abundance gradient (Smartt et al. 2001; Cunha et al. 2007). In addition, the silicate and carbon dust IR bands optical depth per unit magnitude of visual extinction are much stronger in the Galactic center than in the local ISM (Roche \& Aitken 1985; Pendleton et al. 1994) indicating different dust characteristics and thus a different gas-to-dust ratio. Fortunately, one does not have to accurately calculate the hydrogen column, $N(\mathrm{H})$, in order to compute the fractional abundances of $s p^{2}, s p^{3}$, and $\mathrm{H}$ for the observed astronomical hydrocarbons.

The hydrogen content of carbon grains can be measured through the IR absorption features of the aliphatic $\mathrm{CH}_{2}$ and $\mathrm{CH}_{3}$ modes and the aromatic $\mathrm{CH}$ mode. Experimental studies on laboratory materials have shown that these modes have to be carefully unblended-basically because different types of functional groups have intrinsic strengths that can vary by an order of magnitude-but if carefully done, the derived
$\mathrm{H}$-content is in good agreement with other analytical techniques (Ristein et al. 1998). To be consistent with the experimental analysis, for the aliphatic component of the carbon grains, we use the asymmetric $\mathrm{CH}$ stretching modes of the $\mathrm{CH}_{2}$ and $\mathrm{CH}_{3}$ groups (Table 1). We assume that all $s p^{3}$ carbon atoms are bonded to at least one $\mathrm{H}$, so we are ignoring diamond-like structures (materials in the upper apex of the ternary diagram). As the observed interstellar $3.4 \mu \mathrm{m}$ band is characteristic for a:C-H material (see below) and experiments have shown that essentially all $s p^{3}$ sites in a-C:H are hydrogenated (Robertson 2002), this is a reasonable assumption for this work. One measure of the $s p^{2}$ fraction results from the aromatic $\mathrm{CH}$ mode. However, that is only a good measure of the $s p^{2}$ carbon if the $\mathrm{H} / \mathrm{C}$ ratio is high and, consequently, the aromatic domains are small (Jones 2012a). In contrast, the $6.2 \mu \mathrm{m} \mathrm{CC}$ feature is a direct measure of the $\mathrm{C}$ fraction in $s p^{2}$. We have decomposed this latter measure into an olefinic (feature around $1640 \mathrm{~cm}^{-1}$ ) and an aromatic (feature around $1580 \mathrm{~cm}^{-1}$ ) component (see Figure 2; Section 3.1) but for the purpose of the ternary diagram, we have added them together. We note that the measured $\mathrm{H} / \mathrm{C}$ ratio in the aromatic component of interstellar carbon dust is small (Table 1). We take the $\mathrm{CH}_{2}$ and $\mathrm{CH}_{3}$ abundances as our measure of the $s p^{3}$ carbon Hence, we ignore any "pure" C $s p^{3}$ network. Essentially, we are assuming that interstellar carbon has an a-C:H structure rather than a ta-C:H structure (see above). These determinations of the $s p^{2}$ and $s p^{3}$ fractions and the $\mathrm{H}$-content are summarized in Table 2 and shown on the ternary diagram (Figure 3 ). The values derived for the $s p^{2}$ and $s p^{3}$ fractions and the $\mathrm{H}$ content do depend on the adopted intrinsic strength of the relevant vibrational modes. We have adopted values which are entirely consistent with the physical chemistry literature on amorphous hydrocarbon films. As an indication of the systematic uncertainty, somewhat different intrinsic strengths were adopted by Ristein et al. (1998) and this moves the location of interstellar solid carbon on the ternary diagram slightly (Figure 3 ). The values adopted by Jones (2012a) are also very similar. ${ }^{5}$ Earlier studies on interstellar hydrocarbon dust adopted values that were a factor two different (see Section 3.2). As those are not consistent with the location of the hydrocarbon solids in the ternary diagram, we do not consider these here.

The results of our analysis firmly place interstellar carbon dust - on average - as a highly aromatic material (Figure 3). This merely reflects the large carbon column density required to explain the observed $6.2 \mu \mathrm{m}$ band, which is due to an intrinsically very weak $\mathrm{CC}$ mode. The small $\mathrm{H} / \mathrm{C}$ and $s p^{3} / s p^{2}$ ratio derived here for the Galactic center direction are in agreement with studies of local interstellar carbon dust (Pendleton \& Allamandola 2002). In the local ISM, the CC stretch at $6.2 \mu \mathrm{m}$ has not been detected and, indeed, we would not have expected to detect it given the low visual extinction of the sight-lines $\left(A_{V} \leqslant 10 \mathrm{mag}\right)$. The total solid carbon and $s p^{2}$ fraction can, however, be estimated from measured gas phase abundances and the UV extinction curve. Adopting an elemental carbon abundance of $3 \times 10^{-4}$ and an interstellar gas phase $\mathrm{C}$ abundance of $1.5 \times 10^{-4}$ (Cardelli et al. 1996), the ratio of aliphatic $\mathrm{H}$-as measured by the $3.4 \mu \mathrm{m}$ band-to all solid carbon is 0.15 (Pendleton et al. 1994). In the local solar neighborhood, almost all of the carbon in solid form also has to be $s p^{2}$ in order to explain the observed strength of the $2175 \AA$ feature

\footnotetext{
5 Note that the values given in his Table 2 are actually integrated cross sections (A. P. Jones 2012, private communication).
} 
The Lifecycle of Hydrocarbon Dust in the Interstellar Medium

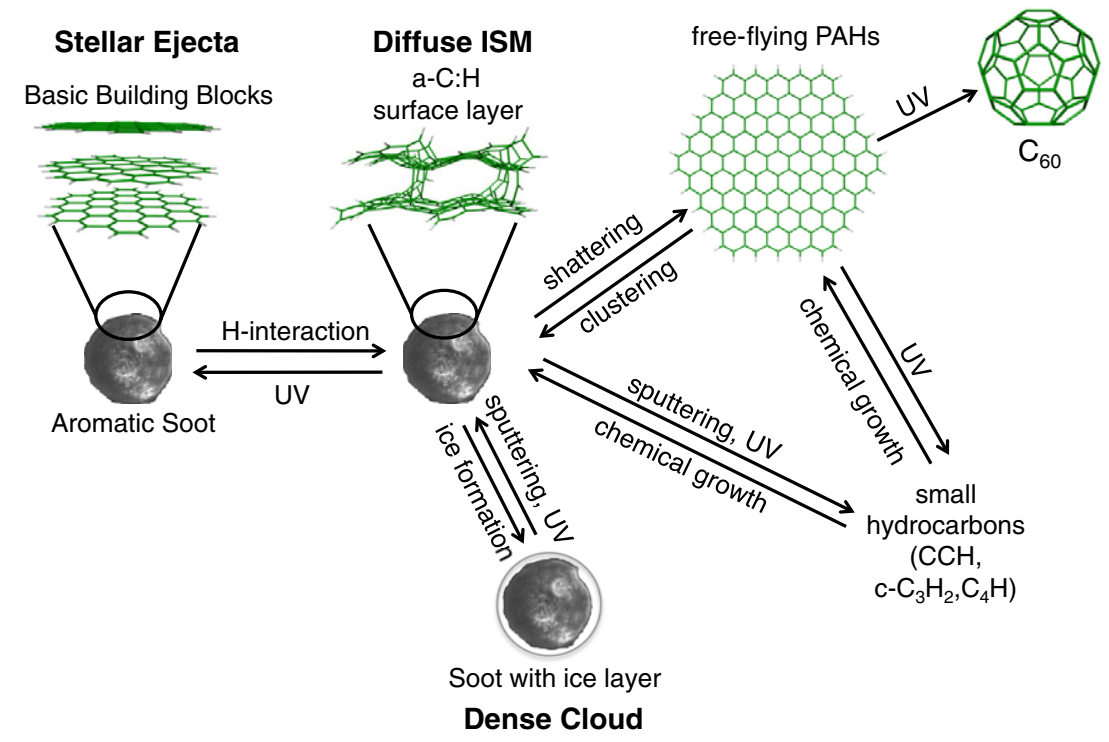

Figure 4. Soot injected by C-rich asymptotic giant branch stars has a highly aromatic character with large graphene sheets stacked into nano grains. Processing by $\mathrm{H}$ atoms and UV photons in the ISM quickly produces a thin $(\sim 10 \AA)$ surface layer of a-C:H material in diffuse interstellar clouds. In dense clouds, this a-C:H layer is lost by photolysis. Shattering by grain-grain collisions in strong shocks in the warm intercloud medium produces free-flying PAH molecules. This shattering is counteracted by clustering in dense cloud environments. Shocks and UV photolysis in the ISM will fragment these PAHs into small hydrocarbons and/or isomerize them into very stable fullerene molecules. Figure adapted from Jones (2009).

(A color version of this figure is available in the online journal.)

(Draine 2003). Assuming that all $s p^{3}$ carbon is in aliphatic $\mathrm{CH}_{2}$ or $\mathrm{CH}_{3}$ groups, the derived $s p^{3} / s p^{2}$ fraction is 0.05 . These numbers are in reasonable agreement with the values derived here for the Galactic center sources (Tables 1 and 2). Dartois et al. (2007), in their pioneering study of interstellar carbon grains, focused on observations of the ULIRG IRAS $08752+3915$ and had only upper limits for the aromatic $\mathrm{CH}$ and the $s p^{2}$ component. Hence, they were forced to use indirect constraints to locate interstellar grains in this source on the ternary plot. These constraints were based upon an evaluation of the structure of $\mathrm{a}-\mathrm{C}: \mathrm{H}$ in terms of random covalent networks. Essentially, this boils down to assuming that the structure of interstellar carbon dust resembles that of hydrogenated amorphous carbon and consists of small aromatic domains connected by aliphatic (and/or olefinic) chains (Jones 1990, 2012a). As our analysis shows, the aromatic $\mathrm{H} / \mathrm{C}$ ratio and the aliphatic/aromatic- $\mathrm{C}$ ratio are very small and, consequently, the aromatic domains are actually very large. Indeed, it has been well recognized that the observed low $\mathrm{H} / \mathrm{C}$ fraction forces us to conclude that the structure of interstellar carbon dust consists of large aromatic moieties connected by small aliphatic chains (Pendleton \& Allamandola 2002) and our analysis presents further observational underpinning of this notion.

The analysis of the IR absorption bands due to interstellar carbon dust represent an average of the dust along the sightline. We recognize, however, that the observed profile of the interstellar $3.4 \mu \mathrm{m}$ band with its detailed substructure dominated by $\mathrm{CH}_{2}$ and $\mathrm{CH}_{3}$ groups in a ratio of $\simeq 2$ (Sandford et al. 1991; Pendleton et al. 1994) is indicative of hydrogenated amorphous carbon, a-C:H, characterized by a relatively high $\mathrm{H} / \mathrm{C}$ ratio $(\simeq 0.5)$. As this inferred microscopic $\mathrm{H} / \mathrm{C}$ ratio of the material carrying the interstellar $3.4 \mu \mathrm{m}$ band is much higher than the average $\mathrm{H} / \mathrm{C}$ ratio derived from our analysis, this is indicative of a two (or even multi) component mixture in a ratio of about 1:3. In principle, these components could be fully independent.
However, we envision the a-C:H as a mantle on top of pure aromatic carbon grains. This core-mantle structure is a natural consequence of the processing of carbon stardust grains by thermal $\mathrm{H}$ atoms, UV photons and/or interstellar shocks in the ISM. We discuss this in some detail in Section 5.

\section{EVOLUTION OF CARBON DUST IN THE ISM}

Figure 4 (adapted from Jones 2009) summarizes the evolution of interstellar carbon dust and the processes that play a role therein. Carbon-rich asymptotic giant branch (AGB) stars inject highly aromatic carbon stardust into the ISM. In the ISM, this carbon stardust is processed by UV photons and thermal $\mathrm{H}$ atoms in diffuse clouds and by implanted protons/H atoms in shock waves, creating a thin surface layer of hydrogenated amorphous carbon material. In shock waves, besides $\mathrm{H}$-implantation, dust will also be sputtered, losing some surface atoms, and shattered through grain-grain collisions, forming PAH molecules. The latter process can be counteracted by coagulation of PAHs in dense clouds. PAH molecules are also lost through UV processing leading to cages, rings, and small (hydro)carbon chains as well as possibly to $\mathrm{C}_{60}$. Depending on the kinetics involved, PAHs may grow through reactions with carbon species. In dense clouds, carbon dust will acquire ice mantles which are quickly removed through photodesorption in the diffuse ISM or through sputtering in frequent, weak shocks. We will discuss each of these aspects in more detail below.

As discussed below, this model for the origin and evolution of interstellar carbon dust will lead to a core-mantle structure where the core consists of aromatic carbon and the surface is hydrogenated. Alternatively, one could consider a model where the two interstellar carbon dust components derive from distinct stellar sources - the main one, C-rich giants producing fully aromatic carbon dust in the low-temperature window (see Section 5.1), with a minor contribution to the interstellar carbon 

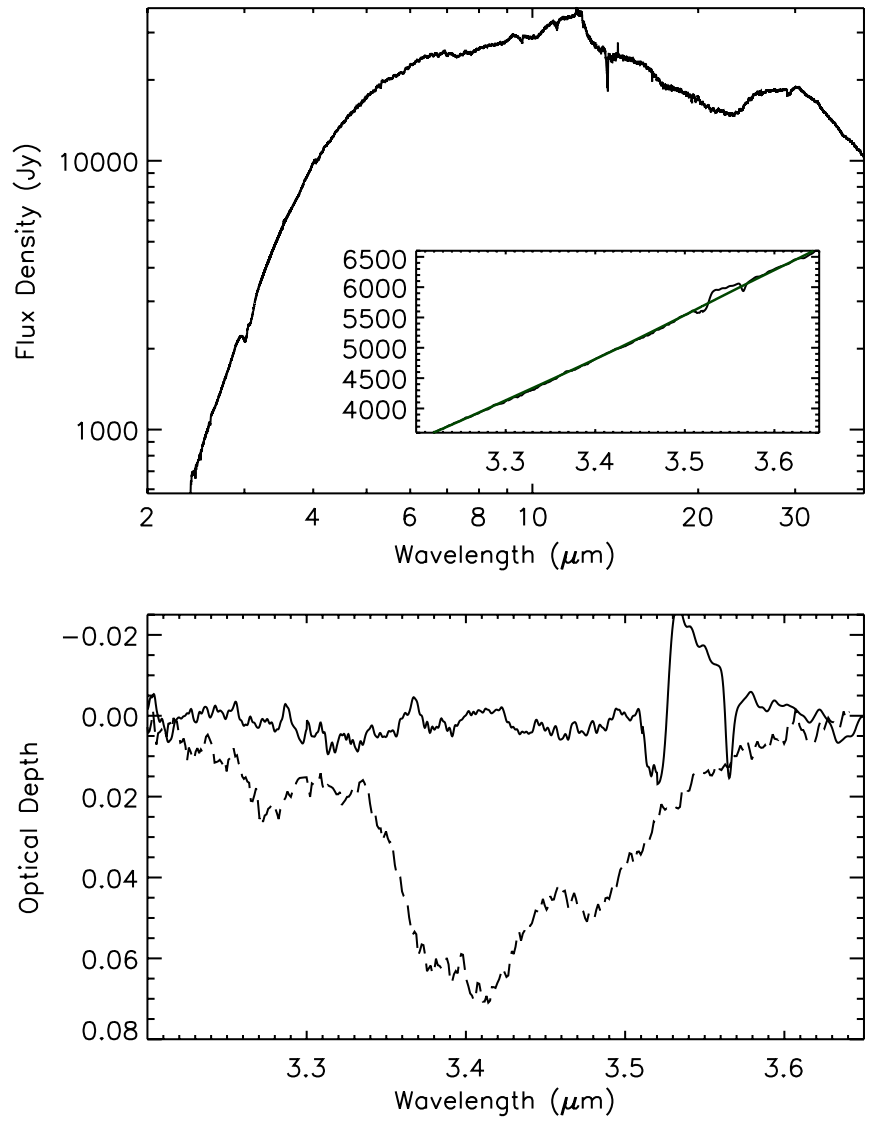

Figure 5. Top panel: ISO-SWS spectrum of IRC+10216. Inset shows wavelength region of $\mathrm{CH}$ stretch in aliphatic hydrocarbons. Overlaid is the polynomial used to create the optical depth spectrum in the panel below. Bottom panel: optical depth spectrum for IRC+10216 (solid line). Superposed is the GC GCS3 spectrum scaled to the depth expected for the $A_{V}$ of IRC+10216 and $\tau / A_{V}=0.004$ for the local ISM (Pendleton et al. 1994).

dust budget in the form of hydrogenated amorphous carbon from a hitherto unidentified stellar source operating in the hightemperature window (see Section 5.1) - and that these dust components are not processed at all in the ISM. However, we deem that inconsistent with our general understanding of the origin and evolution of interstellar dust.

\subsection{Carbon Stardust}

Observational, experimental, and theoretical studies all agree that carbon stardust has a highly aromatic character with a very low H-content. Near-infrared spectroscopy of C-rich AGB stars can be used to place stringent upper limits on the strength of the $3.4 \mu \mathrm{m}$ aliphatic hydrocarbon band. We have analyzed ISO/SWS (from the Sloan et al. 2003 database) of the prototypical C-rich AGB object, IRC+10216 (Figure 5), and two carbon AGB stars (not shown), AFGL 3068 and AFGL 3099, which are representative members of the class of extreme C-stars that dominate the carbon stardust budget of the local ISM. While these objects are very bright at near-infrared wavelengths and hence the ISO/SWS spectra have very high signal-to-noise, the spectra show $\mathrm{C}_{2} \mathrm{H}_{2}$ and $\mathrm{HCN}$ bands around $3.4 \mu \mathrm{m}$ that somewhat hamper detection of the aliphatic bands and we have therefore adopted very conservative local continua to determine $\tau_{3.4}$ as shown for IRC+10216 (Figure 5). The results are summarized in Table 3. The total visual extinctions toward these objects is dominated by circumstellar dust and has been determined through model
Table 3

Hydrogen and Carbon Content in ISM Grains

\begin{tabular}{lcc}
\hline \hline Source & $\begin{array}{c}A_{V} \\
(\mathrm{mag})\end{array}$ & $\tau_{3.4} / A_{V}$ \\
\hline IRC+10216 & $18-27$ & $<0.0003$ \\
AFGL 3068 & $63-86$ & $<0.0005$ \\
AFGL 3099 & $23-30$ & $<0.001$ \\
AFGL 618 & $59-98$ & $0.001-0.002$ \\
Local ISM & $\cdots$ & 0.004 \\
\hline
\end{tabular}

fits to the spectral energy distribution (Martin \& Rogers 1987; Le Bertre 1997). The $3.4 \mu \mathrm{m}$ aliphatic hydrocarbon absorption band has been observed toward the extreme, C-rich post-AGB object, AFGL 618, with an optical depth of 0.1 (Chiar et al. 1998). The visual extinction range, $A_{V}=59-98 \mathrm{mag}$, toward this object has been determined from hydrogen recombination lines in the spectrum. The derived $\tau(3.4 \mu \mathrm{m}) / A_{V}$ ratios (Table 3 ) are much less toward these stardust sources than observed in the local diffuse ISM (Pendleton et al. 1994). It is clear that the $\mathrm{H}$-content and hydrogenated amorphous carbon character of carbon stardust is much less than the already low values derived for ISM dust (Table 2; Section 4). ${ }^{6}$

Analysis of the spectral energy distribution and (infrared) molecular absorption spectra of C-rich AGB stars reveal that the temperature at the inner shell where dust is formed is near 1000 K (Le Bertre 1997; Martin \& Rogers 1987; Fonfría et al. 2008; Keady et al. 1988). This temperature is characteristic for the carbon soot formation window that converts acetylene into large PAH molecules and then soot (Frenklach \& Feigelson 1989; Cherchneff et al. 1992; Cherchneff 2012).

A variety of laboratory studies have focused on the structure of interstellar carbon dust analogs (Mennella et al. 1995, 1996, 2003; Schnaiter et al. 1999; Jäger et al. 2008, 2009; Gadallah et al. 2011). These studies show that carbon soot formed at $\sim 1000 \mathrm{~K}$ has a very high $s p^{2}$ character (>0.9; Jäger et al. 2009; C. Jäger 2012, private communication). The $\mathrm{H}$ content of the soot produced in this low-temperature window has not been quantified, but is very low. The resulting soot grains are highly graphitic, consisting of large graphene layers (up to $3 \mathrm{~nm}$ ). These sizes are comparable to the largest PAH molecules $-\mathrm{C}_{222} \mathrm{H}_{42}$-detected in the MALDI-TOF ${ }^{7}$ studies of these experiments. Soot formation in this temperature window proceeds through the formation of large PAHs and their condensation and further growth onto grains. Experimental studies do reveal a second window of carbon soot formation at much higher temperatures (>1700 K; Jäger et al. 2008, 2009). Soot formed under these conditions has a much higher $\mathrm{H}$-content and a much lower $s p^{2} / s p^{3}$ ratio. However, observations show that carbon dust forms around $\sim 1000 \mathrm{~K}$ (the low temperature window) in C-rich AGB ejecta (see above). In consequence, the higher temperature soot window is of little relevance for the C-star dust budget. It is tempting to speculate that the fullerenes observed to dominate the IR spectra of some, very unique planetary nebulae (e.g., Tc1; Cami et al. 2010) are the byproduct of the soot formation process in this high temperature window but the underlying cause for the opening up of this soot window in these objects is unclear.

\footnotetext{
6 The limit placed on the aliphatic absorption feature is an estimate, and although the emission across this band should be minimal, a proper radiative study would be necessary to firmly quantify the amount of hydrocarbons in these evolved star environments.

7 Matrix-assisted laser desorption/ionization, time of flight.
} 
Some peculiar C-rich post-AGB objects show an appreciable $3.4 \mu \mathrm{m}$ emission band accompanying their PAH emission features. These so-called $21 \mu \mathrm{m}$ objects are low metallicity stars with a mass of $\simeq 1 M_{\odot}$ formed some 10 billion years ago (van Winckel 2003). As a class, these objects have not been very important contributors to the stardust budget over the history of the Milky Way. The peak position and profile of the $3.4 \mu \mathrm{m}$ band in these objects-quite distinct from that of the wellknown $3.4 \mu \mathrm{m}$ band accompanying the $3.3 \mu \mathrm{m}$ interstellar PAH feature $^{8}$ - agrees well with the absorption feature in the ISM and is therefore likely due to aliphatic groups attached as side groups to the PAH skeleton (cf. Joblin et al. 1996). The observed flux in the $3.4 \mu \mathrm{m}$ aliphatic hydrocarbon band is typically comparable to that in the $3.3 \mu \mathrm{m}$ aromatic $\mathrm{CH}$ band. As the intrinsic strength of the former is some 10 times stronger per group than the latter (Joblin et al. 1996), the aliphatic to aromatic character of the emission carrier is still very small $(\sim 0.1)$, even in these types of objects. We emphasize that these $3 \mu \mathrm{m}$ emission features originate from shells at very large distances from the central star (Meixner et al. 1999). The temperature of the dust in these shells is observed to be much too low to allow dust emission at such short wavelengths and the carriers must therefore be molecular in nature (Hony et al. 2003). Hence, these aliphatic groups do not necessarily have any bearing on the character of the carbon dust. Finally, spatially resolved spectroscopy of the 3.3 and $3.4 \mu \mathrm{m}$ features in some of these $21 \mu \mathrm{m}$ objects reveals that the relative strength of the aliphatic to aromatic bands decreases with distance from the central stars (Goto et al. 2003, 2007). As aliphatic $\mathrm{CH}_{2} / \mathrm{CH}_{3}$ groups are more susceptible than aromatic $\mathrm{H}$ to $\mathrm{UV}$ photolysis, this likely reflects processing of the emitting molecules by UV photons from the interstellar radiation field, penetrating the envelope (Joblin et al. 1996). In any case, there is no evidence that the carbon dust released by these objects into the ISM has a high hydrogenated amorphous carbon character. Quite likely, as for the dominant carbon soot factories of the interstellar medium (cf. IRC+10216), in these objects, carbon dust is formed around $1000 \mathrm{~K}$ and is consequently highly aromatic as well.

\subsection{Hydrogenation of Carbon Stardust: H-interaction and UV Photolysis}

In a series of experiments, Mennella and others (Furton \& Witt 1993; Mennella et al. 2001, 2002; Mennella 2006) have investigated the evolution of carbon solids under the influence of a flux of $\mathrm{H}$ atoms and UV photons. The results show that down to the lowest temperature investigated $(80 \mathrm{~K})$, exposure of an aromatic solid to a flux of $\mathrm{H}$ atoms leads to the formation of aliphatic $\mathrm{CH}_{2}$ and $\mathrm{CH}_{3}$ groups until a saturation value is reached. The cross section for this process is $\sigma_{\mathrm{H}} \simeq 1-2 \times 10^{-18} \mathrm{~cm}^{2}$ per $\mathrm{H}$ atom. Under the influence of UV photons, aliphatic $\mathrm{H}$ is lost with a cross section of $\sigma_{\mathrm{UV}} \simeq 10^{-19} \mathrm{~cm}^{2}$ per UV photon. In steady state, the $\mathrm{H} / \mathrm{C}$ coverage, $\theta(\mathrm{H} / \mathrm{C})$, is given by

$$
\theta(\mathrm{H} / \mathrm{C})=\left(1+\frac{\sigma_{\mathrm{UV}} \mathcal{N}_{\mathrm{uv}}}{\sigma_{\mathrm{H}} v_{\mathrm{H}} n_{\mathrm{H}}}\right)^{-1} \theta_{\mathrm{sat}}(\mathrm{H} / \mathrm{C}),
$$

with $\mathcal{N}_{\mathrm{uv}}$ the UV photon flux $\left(10^{8} G_{o}\right.$ photons $\mathrm{cm}^{-2} \mathrm{~s}^{-1}$ with $G_{o}$ the radiation field in units of the Habing average interstellar radiation field), and $v_{H}$ and $n_{\mathrm{H}}$ the atomic $\mathrm{H}$ velocity and density. We take the saturation value, $\theta_{\text {sat }}(\mathrm{H} / \mathrm{C})$, to be 0.5 , typical for a-C:H material. For a typical diffuse cloud with an atomic $\mathrm{H}$

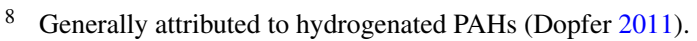

density of $10^{2} \mathrm{~cm}^{-3}$ and a temperature of $100 \mathrm{~K}$, we arrive at a fractional coverage of 0.66 of the saturation value or about 0.3 . As $G_{o} / n_{\mathrm{H}}$ is approximately constant for diffuse clouds in an ISM in pressure and thermal equilibrium, this value will not vary much in the ISM. This steady state value is reached after a time $>10^{4} \mathrm{yr}$. Thus, exposure to atomic $\mathrm{H}$ and UV photons quickly converts a surface layer of aromatic grains into a-C:H material in the ISM. The thickness of this layer will depend on the porosity of the grains but in the experiments the measured saturation absorption strength of $1.6 \times 10^{3} \mathrm{~cm} \mathrm{~g}^{-1}$ corresponds to a $\mathrm{H} / \mathrm{C}$ value averaged over the sample of 0.2 and hence about $1 / 3$ of the sample has been converted into a-C:H material.

The interstellar $3.4 \mu \mathrm{m}$ band has not been observed toward sources behind molecular clouds (Allamandola et al. 1992; Chiar et al. 1996; Brooke et al. 1996), indicating that the balance of hydrogenation and dehydrogenation shifts toward the latter in these environments. Following Mennella et al. (2001), we attribute this to the formation of a thin ice layer on the surfaces of carbon dust grains during the transition from a diffuse cloud to a dense molecular cloud. Mennella et al. (2001) have studied the loss of the aliphatic hydrocarbon band and these experiments show that the $3.4 \mu \mathrm{m}$ band is lost after exposure of (ice-covered) a-C:H grains to a fluence of $3 \times 10^{19}$ photons $\mathrm{cm}^{-2}$. Observations show that ice mantles are formed when the visual extinction exceeds 3 mag (Whittet et al. 2001). For this visual extinction, the UV flux that grains are exposed is $3 \times 10^{13}$ photons $\mathrm{yr}^{-1}$ and the $3.4 \mu \mathrm{m}$ band is lost in $10^{6} \mathrm{yr}$. The timescale for ice formation is $\tau \simeq 3 \times 10^{7} / n_{\mathrm{H}}$ yr per ice layer (see, e.g., Hasegawa \& Herbst 1993; Cazaux et al. 2010). Thus, in $10^{6} \mathrm{yr}$, an ice mantle of some 30 layers is formed at a density of $300 \mathrm{~cm}^{-3}$. Hence, we surmise that these ice layers as well as the conversion of atomic into molecular hydrogen will stop the hydrogenation process.

\section{3. a-C:H Formation by Ion Processing in Interstellar Shocks}

For fluences in excess of $10^{15}$ ions $\mathrm{cm}^{-2}$, shock processing of carbon solids by impinging $\mathrm{H}$ atoms in shock waves will convert aromatic carbon surfaces into hydrogenated amorphous carbon (Tielens et al. 1994 and references therein). For typical interstellar shocks $\left(\simeq 100 \mathrm{~km} \mathrm{~s}^{-1}\right)$, fluences in excess of $10^{19}(a / 1000 \AA)$ ions $\mathrm{cm}^{-2}$ are expected. The estimated range of atomic $\mathrm{H}$ at these energies is some $10 \AA$ or some 5 basal planes. Hence a thin layer of hydrogenated amorphous carbon will be formed with a $\mathrm{H} / \mathrm{C}$ ratio of $\simeq 0.5$ (Tielens et al. 1994). If this process were the main cause of the observed a-C:H component in the diffuse ISM, the observed (average) $\mathrm{H} / \mathrm{C}$ ratio (Table 2) for interstellar grains would imply a typical radius for interstellar carbon grains of $\simeq 50 \AA$.

\subsection{The Destruction of Carbon Grains in Interstellar Shocks}

The rapid destruction of dust grains by interstellar shocks has long been recognized as a key problem for studies of the evolution of interstellar dust (Draine \& Salpeter 1979; Dwek \& Scalo 1980; McKee 1989; Jones et al. 1994, 1996). Theoretical lifetimes for dust grains in the ISM are calculated to be only 400-600 Myr, depending on size and composition. This is much shorter than the injection timescale for stardust into the ISM $\left(\simeq 2 \times 10^{3} \mathrm{Myr}\right)$. This conundrum has been taken to imply that interstellar dust is rapidly reformed in the ISM by (unknown) processes (Tielens 2005; Draine 2009; Jones \& Nuth 2011). There are two aspects (cf. McKee 1989) that enter these theoretical models. (1) A calculation of the destruction of dust grains in interstellar shock waves as a function of grain 
size and material characteristics, and of shock velocity. (2) A convolution of these shock destruction results with a model for the distribution of shock frequencies in the ISM of the Milky Way. Here, we revisit these aspects, focusing on interstellar carbon dust.

Besides implantation and the conversion of graphitic to a-C:H material, impinging $\mathrm{H}$ ions may also sputter some $\mathrm{C}$-atoms from $\mathrm{C}$-grains. As the grain temperatures are rather low $(T \ll 300 \mathrm{~K})$, chemical sputtering is of no concern. Because of its potential as internal lining for tokamak reactors, sputtering of carbon solids is an active area of research and this has been reviewed by Tielens et al. (1994) for astrophysical purposes. Measured yields for $\mathrm{H}$ and $\mathrm{He}$ at the relevant low energies (50-200 eV) are quite low ( $Y_{\text {sput }} \simeq 10^{-2}$ for $\mathrm{H}$ and $Y_{\text {sput }} \simeq 10^{-1}$ for He). It should be understood that as the surfaces of carbon solids are quickly processed to hydrogenated amorphous carbon by the sputtering process, the exact nature and structure of the starting material is not very important. Indeed, they all have similar sputtering yields. The widely used carbon solid yields by Tielens et al. (1994) present a reasonable fit to these data. We note that a recent study by Serra Díaz-Cano \& Jones (2008) arrives at slightly higher sputtering yields (factor of two). Partly, that reflects an adopted mean mass of the sputtered atoms which is in between the $\mathrm{H}$ and $\mathrm{C}$ mass. Such a low mass does not seem warranted. Replacement of a internal $\mathrm{H}$ atom in a a-C:H material by an impinging $\mathrm{H}$ atom does not produce net (carbon) sputtering loss (recall that the sputtering yield per impinging $\mathrm{H}$ atom is much less than unity). The $\mathrm{C}$ binding energy adopted in this study seems also somewhat low and the resulting sputtering yields are high compared to the experimental data available. Hence, in our analysis, we will adopt the destruction rates derived by Jones et al. (1994, 1996), which are based upon the sputtering yields of Tielens et al. (1994).

In each sojourn into the warm intercloud medium, a grain will typically be shocked by one $100 \mathrm{~km} \mathrm{~s}^{-1}$ shock. Theoretical models for sputtering of carbon solids in interstellar shocks lead to the injection of $\simeq 5 \%$ of the solid carbon into the gas phase for such a shock (Jones et al. 1996). Somewhat higher, $\simeq 10 \%$, sputtering losses occur for faster radiative shocks $\left(v_{s} \lesssim 200 \mathrm{~km} \mathrm{~s}^{-1}\right)$ but such shocks are four times less likely to occur.

Observationally, evidence for the destruction of carbon dust in interstellar shocks is scant as the observations are challenging because of the intrinsic weakness of the relevant UV C II absorption line. However, recent observations of the $\mathrm{C}$ II absorption line in the ISM support the idea that the larger grains in dense clouds are fragmented into smaller grains in the low density regions of the neutral ISM (Parvathi et al. 2012). Depletion studies of silicon, magnesium, and iron are much more straightforward. For these species, observations reveal distinct and systematic differences in the level of depletion between the cloud and intercloud phases of the ISM (Savage \& Sembach 1996). As material cycles much more rapidly between the phases of the ISM than it is injected by stars, this depletion pattern reflects a balance of ISM processes; e.g., between shock destruction in the intercloud medium and reaccretion in the denser environment of $\mathrm{H}$ I clouds (Tielens 1998, 2009). The observed large difference in the depletion between these two phases of the ISM, then demonstrates directly that the processes involved-shock destruction and accretion-operate on a timescale similar to the timescale at which material is mixed from the cloud to the intercloud medium and back (Tielens 1998). This mixing timescale is much less ( $\simeq 30$ million years) than the timescale at which new dust is injected into the ISM. Thus, specifically, some $10 \%$ of the iron, $15 \%$ of the magnesium, and some $30 \%$ of the silicon is returned to the gas phase upon each sojourn into the intercloud medium and then rapidly re-accreted once the material is cycled back to the (diffuse) cloud phase. This leads to the formation of a thin, outer skin that protects underlying grain material against destructive processing in shocks (Tielens 1998).

For silicon and magnesium, these differences in depletion between cloud and intercloud phases are slightly larger than theoretical models for sputtering of silicate materials would predict, indicating that the re-accreted material is in the form of mantle with a somewhat lower binding energy than silicates. The depletion pattern of oxygen indicates that it participates in this shock-sputtering and reaccretion cycle. However, carbon does not show a difference in depletion between the cloud and intercloud medium; e.g., the observed variation in the depletion between these phases is less than $10 \%$ (Sofia et al. 2004). This is much less than for silicon, implying that carbon grains are less affected than silicate grains in the ISM by typical $100 \mathrm{~km} \mathrm{~s}^{-1}$ shocks. This is in good agreement with theoretical models for such shock waves (Jones et al. 1996). So, the interstellar depletion studies force us to a model where mantles accreted in the diffuse ISM are oxide-based rather than carbonaceous. We note that the $3.4 \mu \mathrm{m}$ band is not polarized toward the Galactic center while the silicate band is (Adamson et al. 1999; Chiar et al. 2006). Hence, the hydrogenated amorphous carbon dust component does not form a layer on top of silicate grains. This dichotomy between oxide and carbon dust mantles implies that distinct chemical/physical processes dominate their formation and we attribute this to accretion and surface chemistry for the oxides and atomic $\mathrm{H}$ interaction with graphitic grains for the carbonaceous grains.

The destruction of carbon solids can be more important in the hot gas associated with supernova remnants. In those environments, grains are destroyed through thermal sputtering and, theoretical models show that, depending on the grain residence time in the hot gas (e.g., the expansion/rejuvenation of the supernova remnant), an extensive surface layer can be removed (Tielens 2005; Bocchio et al. 2012). Observationally, a large fraction $(60 \%)$ of the solid carbon has been returned to the gas phase in the intermediate and high velocity gas associated with Orion's cloak (Welty et al. 2002). This "cloak" material is associated with the hot bubble in the Orion-Eridanus region and refers to $\mathrm{a} \simeq 100 \mathrm{~km} \mathrm{~s}^{-1}$ shock propagating in a region with a pre-shock density of $\simeq 10^{-3} \mathrm{~cm}^{-3}$ (Welty et al. 2002). Thus, rather than shocking a warm intercloud medium region, these observations refer to a shock propagating into hot intercloud medium gas and reflects probably the rejuvenation of a preexisting supernova remnant by a new supernova, where it should be understood that the current velocity of the gas is only a lower limit to the shock velocity as the shocked gas may have been slowed down by now (Cowie 1978). Curiously, while carbon dust has been largely destroyed, much less silicon and iron $(\simeq 10 \%)$ has been returned to the gas phase (Welty et al. 2002). Gas associated with the so-called local interstellar cloud near the Sun-actually also low density, intercloud material but now inside the local bubble-also indicates that interstellar carbon dust has been much more destroyed than silicates in this environment (Slavin \& Frisch 2006). Thus, while observations show that low velocity, radiative shocks into warm intercloud medium material destroy more silicates than carbon dust, exposure to hot gas inside a supernova remnant seems to have the opposite effect. For thermal sputtering in hot gas, grains 
of different sizes lose the same thickness surface layer. These differences between destruction in the warm and hot phases of the ISM might be reconciled if carbon dust grains are much smaller than silicate grains. Small sizes $(\simeq 50 \AA)$ coupled with their low specific density ensure that carbon dust is rapidly stopped in radiative shocks and faces little destruction while large, and denser, silicate grains are betatron accelerated and suffer much larger sputtering losses (Jones et al. 1994). In hot gas that fully erodes $50 \AA$ carbon grains, the fractional destruction of large silicate grains could still be small $(3 \Delta a / a \simeq 0.1$ for $\Delta a=50 \AA$ and $a=1500 \AA$ ).

The total mass of hot intercloud medium gas in the Milky Way is some $10^{8} M_{\odot}$ but in the plane this is only some $M_{\mathrm{HIM}} \simeq 10^{7} M_{\odot}$. Typically, $\mathrm{OB}$ associations form massive stars over an $\Delta \tau_{\mathrm{OB}}=10-20 \mathrm{Myr}$ time interval. Moreover, the lifetime of the lowest mass $\left(8 M_{\odot}\right)$ core collapse progenitor is some $40 \mathrm{Myr}$. For example, the age spread of the Orion OB association subgroups-responsible for the Orion-Eridanus superbubble-is in excess of $10 \mathrm{Myr}$ and the youngest subgroups are still forming (Brown et al. 1994; Blaauw 1991). Essentially, after the first core collapse supernova explosion, subsequent ones merely rejuvenate the superbubble and their energy is expended in driving the (slow) expansion of swept-up ISM (Shull \& Saken 1995). However, the shocks associated with this expansion are too slow to contribute to processing interstellar dust (McKee 1989). The timescale for reprocessing all of the ISM through superbubbles is then $\left(M_{\mathrm{ISM}} / M_{\mathrm{HIM}}\right) \Delta \tau_{\mathrm{OB}} \simeq(7 \times$ $\left.10^{9} / 10^{7}\right) 10^{7}=7 \times 10^{9} \mathrm{yr}$. Hence, while hot gas in superbubbles may destroy most of their associated carbon dust, on a galactic scale they are of little importance for the dust destruction budget.

Runaway OB stars and supernovae Type Ia may explode outside of such superbubbles. Censuses of local OB stars show that some $10 \%-20 \%$ of all OB stars may be ejected from their association and explode in the field (Gvaramadze et al. 2011; Bragança et al. 2012; Fujii \& Portegies Zwart 2011). Analysis of a variety of galactic and extragalactic data shows that some $80 \%-90 \%$ of the core collapse supernovae explode within superbubbles and only a small fraction explode in the field (Higdon \& Lingenfelter 1980, 2005). This is not too surprising as B stars are the predominant core collapse supernova progenitors and only a very small fraction (a few percent) end up as runaways. Based on galactic chemical evolution models, the ratio of Type Ia to core collapse supernovae is $15 \%$ over cosmic timescales (Tsujimoto et al. 1995) or about $3 \times 10^{-3} \mathrm{yr}^{-1}$. The Type Ia supernova rate for $\mathrm{Sbc}$ galaxies is measured to be $\simeq 1.1 \times 10^{-13}$ per unit stellar mass (Li et al. 2011). With a stellar mass of $6 \times 10^{10} M_{\odot}$, this yields a Type Ia supernova rate of $7 \times 10^{-3}$ for the Milky Way. Here, for convenience, we will adopt the former. In addition to their low frequency, the runaway Type II supernova and Type Ia supernova may be much less effective in destroying interstellar dust as they tend to explode well above the gas disk and this may amount to factor three in the effective supernova rate (McKee 1989). Taking an overall supernova rate of $2 \times 10^{-2}$ for the Milky Way of which 0.15 are runaway OB stars or Type Ia supernova and 0.33 of those occur in the gas disk, we arrive at an effective supernova rate of $10^{-3}$; almost an order of magnitude less than adopted by McKee (1989). Such supernovae would process $\sim 300 M_{\odot}$ of ISM material in the hot gas and destroy all the dust therein. The resulting lifetime of dust against destruction by these supernovae is then $\left(M_{\mathrm{ISM}} / M_{\mathrm{SNR}}\right) k_{\mathrm{eff}}^{-1} \simeq\left(7 \times 10^{9} / 300\right) 10^{3} \simeq 2 \times 10^{10} \mathrm{yr}$.

Summarizing this discussion, we conclude that observational and theoretical evidence shows that low velocity, radia- tive shocks $\left(<250 \mathrm{~km} \mathrm{~s}^{-1}\right)$ - the predominant shocks in the ISM-are not effective in destroying interstellar carbon dust. Carbon dust is effectively processed in the hot gas associated with supernova remnants (e.g., non-radiative fast shocks). However, little interstellar gas is processed by such regions as supernova energy is mainly expended in rejuvenating existing supernovae, eventually blowing superbubbles that vent into the lower halo. As an aside, we note that silicate dust is more effectively processed by low velocity shocks but the sputtered material is rapidly re-accreted into an (oxide) mantle, protecting the underlying core against further processing (Tielens 2005, 2009). Hence, neither the carbonaceous grains nor the silicate grains are very effectively destroyed by supernova shocks.

\subsection{Carbon Dust Growth in the ISM}

There is indirect observational evidence for accretion of mantles in the diffuse ISM in the variation of the depletion pattern of silicon, magnesium, iron and other refractory elements between diffuse clouds and the intercloud medium. However, carbon does not participate in this depletion pattern (cf. Section 5.4). This has several implications for carbon solids. First, as we recognized in Section 5.4, carbon solids are not readily destroyed in the typical shocks in the warm intercloud medium. Second, as there is a large reservoir of gaseous carbon available in diffuse clouds, accretion and mantle formation in the diffuse ISM does not involve carbon. Hence, the re-accreted thin outer layer of interstellar dust has an oxide rather than a carbide structure. This difference in chemical behavior between carbon and, e.g., silicon is not understood: perhaps, accreted carbon is rapidly cycled to volatile compounds (e.g., $\mathrm{CH}_{4}, \mathrm{CO}$ ) that are readily photodesorbed rather than become integrated into a carbide, silicate, or oxide network (Tielens 2009).

Carbon solid formation is perhaps possible in dense molecular clouds. In the transition from diffuse atomic clouds to molecular clouds, the gas will go through a phase lasting some $10^{6} \mathrm{yr}$ in which small hydrocarbon radicals and acetylene derivatives are abundant. During this so-called early time chemistry, the predominant form of carbon in diffuse clouds, $\mathrm{C}^{+}$, is converted into the dominant carbon bearing species in molecular clouds, $\mathrm{CO}$, and hydrocarbon radicals and acetylene derivatives are the molecular intermediaries. It is possible that these species react with PAHs and carbon solids through barrier-less neutral-neutral reactions (Parker et al. 2012). This reaction pathway for carbon dust growth may be assisted by the prevalent FUV photons present during this transition phase, creating radical sites on dust grain surfaces. However, this chemical route toward carbon dust growth has not been quantified.

Past models for the growth of carbonaceous solid material in dense molecular clouds have invariably focused on carbonaceous mantles formed by energetic processing (e.g., UV photolysis or ion bombardment) of simple molecular ice mixtures. There is ample experimental evidence for the importance of this process in laboratory settings (Greenberg et al. 1995; Allamandola et al. 1999; Schutte et al. 1992). However, there is no observational evidence for the importance of energetic processing of simple ices in the ISM. In this context, residues produced by photolysis of ice mantles-containing the dominant interstellar ice molecules, $\mathrm{H}_{2} \mathrm{O}, \mathrm{CH}_{3} \mathrm{OH}$, $\mathrm{NH}_{3}$, and $\mathrm{CO}$-invariably produces absorption in the 4 to $8 \mu \mathrm{m}$ region due to nitrogen and oxygen bearing compounds that are much stronger than the $3.4 \mu \mathrm{m}$ hydrocarbon bands. These strong, longer wavelength bands are not observed in the 
infrared absorption spectrum of dust in the diffuse ISM (Schutte et al. 1998; Chiar et al. 2000; Pendleton \& Allamandola 2002; Dartois et al. 2005). Moreover, the radical HCO is readily formed in photolyzed mixtures of $\mathrm{H}_{2} \mathrm{O}$ and $\mathrm{CO}$ ice; however, its distinctive carbonyl mode at $5.6 \mu \mathrm{m}$ has never been observed in interstellar ice spectra (Tielens 2013). Similarly, the organic inventory of hot cores-derived from evaporating interstellar ices near the newly formed star-is at odds with energetic processing of interstellar ices. Indeed, photolysis of ices will lead to a wide range of simple molecular products, much in contrast to hot cores that show much chemical specificity (Tielens 2013). The latter two arguments suggest that the UV field inside dense clouds is less than assumed in these laboratory-analog studies.

\subsection{PAHs and Carbon Stardust: Shattering and Coagulation}

Theoretical studies of processing of interstellar PAHs by shock waves and UV photons in the ISM yield very short lifetimes, of the order of $10^{8} \mathrm{yr}$ (Micelotta et al. 2010a, 2010b). While this lifetime is somewhat uncertain as the relevant binding energy of carbon is not well known for astrophysical relevant PAHs, this timescale is quite short compared to the injection timescale of PAHs by C-rich AGB stars. Hence, as some $10 \%$ of the elemental $\mathrm{C}$ is locked up in interstellar PAHs, there must be rapid replenishment process in the ISM. One such process could be shattering by grain-grain collisions in low velocity interstellar shocks (Jones et al. 1996). This is a very attractive process given the highly aromatic nature and the presence of large graphene sheets in carbon soot (Jäger et al. 2009). This process will be counteracted by sputtering of the resulting PAH fragments in the shock which is calculated to be important for shock velocities of $100 \mathrm{~km} \mathrm{~s}^{-1}$ or larger (Micelotta et al. 2010a). We note however that during each sojourn of a dust grain in the warm neutral medium it will see some four $50 \mathrm{~km} \mathrm{~s}^{-1}$ shocks as compared to only one $100 \mathrm{~km} \mathrm{~s}^{-1}$ shock. So, shattering may still be important as a source of interstellar PAHs.

Actually, calculations show that shattering is very efficient, completely breaking down a majority of the carbon dust into small fragments even for a shock velocity of $50 \mathrm{~km} \mathrm{~s}^{-1}$ (Jones et al. 1996) and perhaps even lower. Within this context, shattering of carbon dust must be counteracted by rapid coagulation in cloud environments. Joblin and coworkers (Rapacioli et al. 2005; Berné et al. 2007) have analyzed Spitzer IRS spectra of photodissociation regions and concluded that the smallest carbon dust grains consist of clustered PAHs. These so-called very small grains are so small ( $\simeq 300 \mathrm{C}$-atoms) that they evaporate into their constituent PAH molecules when exposed to strong UV fields (Rapacioli et al. 2006). Larger PAH clusters would, however, be stable against UV-driven evaporation. With $10 \%$ of the elemental $\mathrm{C}$ locked up in PAHs, the total cross section of PAHs is $10^{-21} \mathrm{~cm}^{2} / \mathrm{H}$ atom. As most collisions are expected to lead to effective growth, the clustering rate is $\simeq 6 \times 10^{-18} n_{\mathrm{H}} \mathrm{s}^{-1}$ in diffuse clouds. If all the solid carbon $\left(\simeq 1.5 \times 10^{-4}\right.$ per $\mathrm{H}$ atom) is in the form of small fragments, this timescale would be a factor of five faster. With the former number, a clustering collision takes about $25 \mathrm{Myr}$ at a density $10^{2} \mathrm{~cm}^{-3}$. So, for clustering to be effective in building up larger structures requires long timescales $(\simeq 100 \mathrm{Myr})$ in dense environments $\left(10^{3} \mathrm{~cm}^{-3}\right)$. These values seem somewhat prohibitive and it is unclear whether a balance between coagulation and shattering is possible, but perhaps the fragmentation efficiency is less than theoretically estimated.

\section{SUMMARY}

We have used new and previously published spectra of the aromatic and aliphatic hydrocarbon stretching vibrations to assess the structure of hydrocarbon dust in the diffuse ISM. For the line of sight that probes $A_{V} \sim 29 \mathrm{mag}$ of diffuse ISM dust along the line of sight toward the Galactic center Quintuplet Cluster, the aromatic $3.28 \mu \mathrm{m} \mathrm{CH}$ and $6.2 \mu \mathrm{m} \mathrm{CC}$, in addition to the well-studied $3.4 \mu \mathrm{m}$ aliphatic $\mathrm{CH}$ stretching modes, are all detected. Careful decomposition of the various modes and use of experimentally determined absorption strengths appropriate for hydrocarbon films allow us to use these features to calculate the fractional $s p^{2}, s p^{3}$, and $\mathrm{H}$ content in the carbonaceous grains. The structure of the diffuse ISM hydrocarbons can be understood by use of the ternary diagram which describes these fractional abundances in graphic form. The low $\mathrm{H}$ and high aromatic content (large aromatic domains) of the diffuse ISM carbonaceous dust result in the diffuse ISM point being located near the $s p^{2}$ apex along the bottom edge of the diagram.

We have placed the results of our analysis within the context of the evolution of carbon dust in the ISM. In our view, interstellar carbon dust consists of a large core of aromatic carbon surrounded by a thin layer of hydrogenated amorphous carbon. This structure is a natural consequence of the processing of stardust grains in the ISM. Analysis of IR observations of the main, stellar, carbon dust sources reveals that carbon stardust injected into the ISM has a highly aromatic character and consists mainly of large graphene sheets with very little aliphatic or aromatic hydrogen. This is in good agreement with experimental and theoretical studies. The surface layers of carbon stardust are then quickly hydrogenated by the action of thermal $\mathrm{H}$ atoms in diffuse interstellar clouds. This process is counteracted by UV photolysis. In the diffuse ISM, the balance of these processes will be lead to a hydrogenated amorphous carbon surface structure with a high $\mathrm{H} / \mathrm{C}(\simeq 0.5)$ ratio. In dense clouds, on the other hand, the surface will revert back to a fully aromatic structure. In any case, only a small surface layer of the grains is affected by these processes. We have also reexamined observational and theoretical evidence for carbon dust destruction by strong shocks and conclude that low velocity $\left(<200 \mathrm{~km} \mathrm{~s}^{-1}\right)$ shocks are not very effective in destroying carbon dust. High velocity $\left(\gg 200 \mathrm{~km} \mathrm{~s}^{-1}\right)$, nonradiative shocks are much more prone to destroy carbon dust but only a very small fraction of the ISM is processed by supernovae at these kinds of velocities. Overall, the lifetime of carbon stardust against sputtering in shocks is expected to be very long $\left(>7 \times 10^{9} \mathrm{yr}\right)$. Finally, we examine the interrelationship between carbon stardust and PAH molecules. Shattering collisions of carbon stardust grains in strong shock waves may be an important source of interstellar PAHs, but it is unclear whether clustering of PAHs can reform these clusters again in the diffuse ISM.

This work was supported by NASA's Long Term Space Astrophysics Program (399-20-61-02). This work was initiated while J.E.C. was a visiting scientist at the Joint Astronomy Centre to whom the author is grateful for support. The United Kingdom Infrared Telescope is operated by the Joint Astronomy Centre on behalf of the UK Particle Physics and Astronomy Research Council. Studies of interstellar PAHs and interstellar dust at Leiden Observatory are supported through advancedERC grant 246976 from the European Research Council, through a grant by the Dutch Science Agency, NWO, as part 
of the Dutch Astrochemistry Network, and through the Spinoza premie from the Dutch Science Agency, NWO. A.R. thanks NASA Astrophysics Theory and Fundamental Physics (ATFP; NNX09AD18G) program for the generous support of this work. We thank the referee, Ant Jones, for his thoughtful review that greatly improved the paper.

Facilities: UKIRT (CGS4), Spitzer (IRS)

\section{REFERENCES}

Adamson, A. J., Whittet, D. C. B., Chrysostomou, A., et al. 1999, ApJ, 512, 224 Adamson, A. J., Whittet, D. C. B., \& Duley, W. W. 1990, MNRAS, 243, 400

Allamandola, L. J., Bernstein, M. P., Sandford, S. A., \& Walker, R. L. 1999, SSRv, 90, 219

Allamandola, L. J., Sandford, S. A., Tielens, A. G. G. M., \& Herbst, T. M. 1992, ApJ, 399, 134

Armus, L., Charmandaris, V., Bernard-Salas, J., et al. 2007, ApJ, 656, 148

Berné, O., Joblin, C., Deville, Y., et al. 2007, A\&A, 469, 575

Bernstein, M. P., Sandford, S. A., \& Allamandola, L. J. 2005, ApJS, 161, 53

Blaauw, A. 1991, in NATO ASIC Proc. 342, The Physics of Star Formation and Early Stellar Evolution, ed. C. J. Lada \& N. D. Kylafis (Dordrecht: Kluwer), 125

Bocchio, M., Micelotta, E. R., Gautier, A.-L., \& Jones, A. P. 2012, A\&A, 545, A124

Bourée, J., Godet, C., Etemadi, R., \& Drévillon, B. 1996, Synth. Met., 76, 191 Bragança, G. A., Daflon, S., Cunha, K., et al. 2012, AJ, 144, 130

Brooke, T. Y., Sellgren, K., \& Geballe, T. R. 1999, ApJ, 517, 883

Brooke, T. Y., Sellgren, K., \& Smith, R. G. 1996, ApJ, 459, 209

Brown, A. G. A., de Geus, E. J., \& de Zeeuw, P. T. 1994, A\&A, 289, 101

Butchart, I., McFadzean, A. D., Whittet, D. C. B., Geballe, T. R., \& Greenberg, J. M. 1986, A\&A, 154, L5

Cami, J., Bernard-Salas, J., Peeters, E., \& Malek, S. E. 2010, Sci, 329, 1180

Cardelli, J. A., Meyer, D. M., Jura, M., \& Savage, B. D. 1996, ApJ, 467, 334

Cazaux, S., Cobut, V., Marseille, M., Spaans, M., \& Caselli, P. 2010, A\&A, 522, A74

Cherchneff, I. 2012, A\&A, 545, A12

Cherchneff, I., Barker, J. R., \& Tielens, A. G. G. M. 1992, ApJ, 401, 269

Chiar, J. E., Adamson, A. J., Pendleton, Y. J., et al. 2002, ApJ, 570, 198

Chiar, J. E., Adamson, A. J., \& Whittet, D. C. B. 1996, ApJ, 472, 665

Chiar, J. E., Adamson, A. J., Whittet, D. C. B., et al. 2006, ApJ, 651, 268

Chiar, J. E., Pendleton, Y. J., Geballe, T. G., \& Tielens, A. G. G. M. 1998, ApJ, 507,281

Chiar, J. E., Tielens, A. G. G. M., Whittet, D. C. B., et al. 2000, ApJ, 537, 749

Choi, S., Lee, K.-R., Oh, S., \& Lee, S. 2001, ApSS, 169, 217

Cowie, L. L. 1978, ApJ, 225, 887

Cunha, K., Sellgren, K., Smith, V. V., et al. 2007, ApJ, 669, 1011

Dartois, E., Geballe, T. R., Pino, T., et al. 2007, A\&A, 463, 635

Dartois, E., \& Muñoz-Caro, G. M. 2007, A\&A, 476, 1235

Dartois, E., Muñoz-Caro, G. M., Deboffle, D., Montagnac, G., \& D'Hendecourt, L. 2005, A\&A, 432, 895

Dischler, B. 1983, SSCom, 48, 105

Dopfer, O. 2011, in EAS Publications Series, Vol. 46, PAHs and the Universe: A Symposium to Celebrate the 25th Anniversary of the PAH Hypothesis, ed. C. Joblin \& A. G. G. M. Tielens (Les Ulis: EDP Sciences), 103

Draine, B. T. 2003, ARA\&A, 41, 241

Draine, B. T. 2009, in ASP Conf. Ser. 414, Cosmic Dust-Near and Far, ed. T. Henning, E. Grün, \& J. Steinacker (San Francisco, CA: ASP), 453

Draine, B. T., \& Salpeter, E. E. 1979, ApJ, 231, 438

Duley, W. W. 1984, ApJ, 287, 694

Duley, W. W. 1994, ApJL, 430, L133

Duley, W. W., Scott, A. D., Seahra, S., \& Dadswell, G. 1998, ApJL, 503, L183

Duley, W. W., \& Williams, D. A. 1983, MNRAS, 205, 67P

Dwek, E., \& Scalo, J. M. 1980, ApJ, 239, 193

Ferrari, A. C., \& Robertson, J. 2000, PhRvB, 61, 14095

Figer, D. F., McLean, I. S., \& Morris, M. 1999, ApJ, 514, 202

Fonfría, J. P., Cernicharo, J., Richter, M. J., \& Lacy, J. H. 2008, ApJ, 673, 445

Frenklach, M., \& Feigelson, E. D. 1989, ApJ, 341, 372

Fujii, M. S., \& Portegies Zwart, S. 2011, Sci, 334, 1380

Furton, D. G., Laiho, J. W., \& Witt, A. N. 1999, ApJ, 526, 752

Furton, D. G., \& Witt, A. N. 1993, ApJL, 415, L51

Gadallah, K. A. K., Mutschke, H., \& Jäger, C. 2011, A\&A, 528, A56

Gielen, J. W. A. M., Kleuskens, P. R. M., van de Sanden, M. C. M., et al. 1996, JAP, 80, 5986

Goto, M., Gaessler, W., Hayano, Y., et al. 2003, ApJ, 589, 419

Goto, M., Kwok, S., Takami, H., et al. 2007, ApJ, 662, 389
Greenberg, J. M., Li, A., Mendoza-Gomez, C. X., et al. 1995, ApJL, 455, L177 Gvaramadze, V. V., Kniazev, A. Y., Kroupa, P., \& Oh, S. 2011, A\&A, 535, A29 Hasegawa, T. I., \& Herbst, E. 1993, MNRAS, 263, 589

Heitz, T., Drévillon, B., Godet, C., \& Bourée, J. E. 1998, PhRvB, 58, 13957

Higdon, J. C., \& Lingenfelter, R. E. 1980, ApJ, 239, 867

Higdon, J. C., \& Lingenfelter, R. E. 2005, ApJ, 628, 738

Hony, S., Tielens, A. G. G. M., Waters, L. B. F. M., \& de Koter, A. 2003, A\&A, 402, 211

Imanishi, M. 2000, MNRAS, 319, 331

Imanishi, M., Dudley, C. C., \& Maloney, P. R. 2006, ApJ, 637, 114

Jacob, W., \& Möller, W. 1993, ApPhL, 63, 1771

Jäger, C., Huisken, F., Mutschke, H., Jansa, I. L., \& Henning, T. 2009, ApJ, 696, 706

Jäger, C., Mutschke, H., Henning, T., \& Huisken, F. 2008, ApJ, 689, 249

Joblin, C., Boissel, P., Leger, A., d'Hendecourt, L., \& Defourneau, D. 1995, A\&A, 299, 835

Joblin, C., Tielens, A. G. G. M., Allamandola, L., \& Geballe, T. R. 1996, ApJ, 458, 610

Jones, A. P. 1990, MNRAS, 247, 305

Jones, A. P. 2009, in ASP Conf. Ser. 414, Cosmic Dust-Near and Far, ed. T. Henning, E. Grün, \& J. Steinacker (San Francisco, CA: ASP), 473

Jones, A. P. 2012a, A\&A, 540, A1

Jones, A. P. 2012b, A\&A, 540, A2

Jones, A. P. 2012c, A\&A, 542, A98

Jones, A. P., \& Nuth, J. A. 2011, A\&A, 530, A44

Jones, A. P., Tielens, A. G. G. M., \& Hollenbach, D. J. 1996, ApJ, 469, 740

Jones, A. P., Tielens, A. G. G. M., Hollenbach, D. J., \& McKee, C. F. 1994, ApJ, 433, 797

Keady, J. J., Hall, D. N. B., \& Ridgway, S. T. 1988, ApJ, 326, 832

Kondo, T., Kaneda, H., Oyabu, S., et al. 2012, ApJL, 751, L18

Le Bertre, T. 1997, A\&A, 324, 1059

Lequeux, J., \& Jourdain de Muizon, M. 1990, A\&A, 240, L19

Li, W., Chornock, R., Leaman, J., et al. 2011, MNRAS, 412, 1473

Martin, P. G., \& Rogers, C. 1987, ApJ, 322, 374

Mason, R. E., Wright, G., Pendleton, Y., \& Adamson, A. 2004, ApJ, 613, 770

Mathis, J. S., Mezger, P. G., \& Panagia, N. 1983, A\&A, 128, 212

McFadzean, A. D., Whittet, D. C. B., Bode, M. F., Adamson, A. J., \& Longmore, A. J. 1989, MNRAS, 241, 873

McKee, C. F. 1989, ApJ, 345, 782

Meixner, M., Ueta, T., Dayal, A., et al. 1999, ApJS, 122, 221

Mennella, V. 2006, ApJL, 647, L49

Mennella, V., Baratta, G. A., Esposito, A., Ferini, G., \& Pendleton, Y. J. 2003, ApJ, 587, 727

Mennella, V., Brucato, J. R., Colangeli, L., \& Palumbo, P. 2002, ApJ, 569, 531

Mennella, V., Colangeli, L., Bussoletti, E., et al. 1995, P\&SS, 43, 1217

Mennella, V., Colangeli, L., Palumbo, P., et al. 1996, ApJL, 464, L191

Mennella, V., Muñoz Caro, G. M., Ruiterkamp, R., et al. 2001, A\&A, 367, 355

Micelotta, E. R., Jones, A. P., \& Tielens, A. G. G. M. 2010a, A\&A, 510, A37

Micelotta, E. R., Jones, A. P., \& Tielens, A. G. G. M. 2010b, A\&A, 510, A36

Moneti, A., Cernicharo, J., \& Pardo, J. R. 2001, ApJL, 549, L203

Parker, D. S. N., Zhang, F., Kim, Y. S., et al. 2012, PNAS, 109, 53

Parvathi, V. S., Sofia, U. J., Murthy, J., \& Babu, B. R. S. 2012, ApJ, 760, 36

Pendleton, Y. J., \& Allamandola, L. J. 2002, ApJS, 138, 75

Pendleton, Y. J., Sandford, S. A., Allamandola, L. J., Tielens, A. G. G. M., \& Sellgren, K. 1994, ApJ, 437, 683

Rapacioli, M., Calvo, F., Joblin, C., et al. 2006, A\&A, 460, 519

Rapacioli, M., Joblin, C., \& Boissel, P. 2005, A\&A, 429, 193

Ristein, J., Stief, R. T., Ley, L., \& Beyer, W. 1998, JAP, 84, 3836

Robertson, J. 2002, MSEnR, 37, 129

Roche, P. F., \& Aitken, D. K. 1985, MNRAS, 215, 425

Sandford, S. A., Allamandola, L. J., Tielens, A., et al. 1991, ApJ, 371, 607

Savage, B. D., \& Sembach, K. R. 1996, ARA\&A, 34, 279

Schnaiter, M., Henning, T., Mutschke, H., et al. 1999, ApJ, 519, 687

Schutte, W. A., Allamandola, L. J., \& Sandford, S. A. 1992, AdSpR, 12, 47

Schutte, W. A., van der Hucht, K. A., Whittet, D. C. B., et al. 1998, A\&A, 337,261

Sellgren, K. 2001, AcSpe, 57, 627

Serra Díaz-Cano, L., \& Jones, A. P. 2008, A\&A, 492, 127

Shull, J. M., \& Saken, J. M. 1995, ApJ, 444, 663

Slavin, J. D., \& Frisch, P. C. 2006, ApJL, 651, L37

Sloan, G. C., Kraemer, K. E., Price, S. D., \& Shipman, R. F. 2003, ApJS, 147,379

Smartt, S. J., Venn, K. A., Dufton, P. L., et al. 2001, A\&A, 367, 86

Sofia, U. J., Lauroesch, J. T., Meyer, D. M., \& Cartledge, S. I. B. 2004, ApJ, 605,272

Spoon, H. W. W., Armus, L., Cami, J., et al. 2004, ApJS, 154, 184 
Spoon, H. W. W., Keane, J. V., Tielens, A. G. G. M., Lutz, D., \& Moorwood, A. F. M. 2001, A\&A, 365, L353

Tanaka, M., Sato, S., Nagata, T., \& Yamamoto, T. 1990, ApJ, 352, 724

Tielens, A. G. G. M. 1998, ApJ, 499, 267

Tielens, A. G. G. M. 2005, The Physics and Chemistry of the Interstellar Medium (Cambridge: Cambridge Univ. Press)

Tielens, A. G. G. M. 2009, in EAS Publications Series, Vol. 35, Astrophysics in the Next Decade, ed. H. A. Thronson, M. Stiavelli, \& A. Tielens (Les Ulis: EDP Sciences), 33

Tielens, A. G. G. M. 2013, RvMP, in press

Tielens, A. G. G. M., McKee, C. F., Seab, C. G., \& Hollenbach, D. J. 1994, ApJ, 431,321
Tielens, A. G. G. M., Wooden, D. H., Allamandola, L. J., Bregman, J., \& Witteborn, F. C. 1996, ApJ, 461, 210

Tsujimoto, T., Nomoto, K., Yoshii, Y., et al. 1995, MNRAS, 277, 945

van Winckel, H. 2003, ARA\&A, 41, 391

Welty, D. E., Jenkins, E. B., Raymond, J. C., Mallouris, C., \& York, D. G. 2002, ApJ, 579, 304

Wexler, A. S. 1967, ApSRv, 1, 29

Whittet, D. C. B., Boogert, A. C. A., Gerakines, P. A., et al. 1997, ApJ, 490, 729

Whittet, D. C. B., Gerakines, P. A., Hough, J. H., \& Shenoy, S. S. 2001, ApJ, 547,872 